\title{
Estimation and prediction for spatial generalized linear mixed models with parametric links via reparameterized importance sampling
}

\author{
Evangelos Evangelou ${ }^{1}$ and Vivekananda Roy ${ }^{2}$ \\ ${ }^{1}$ Department of Mathematical Sciences, University of Bath, Bath, BA2 7AY, UK. \\ 2 Department of Statistics, Iowa State University, 3415 Snedecor Hall, Ames, IA, USA.
}

January 9, 2019

\begin{abstract}
Spatial generalized linear mixed models (SGLMMs) are popular for analyzing non-Gaussian spatial data. These models assume a prescribed link function that relates the underlying spatial field with the mean response. There are circumstances, such as when the data contain outlying observations, where the use of a prescribed link function can result in poor fit, which can be improved by using a parametric link function. Some popular link functions, such as the Box-Cox, are unsuitable because they are inconsistent with the Gaussian assumption of the spatial field. We present sensible choices of parametric link functions which possess desirable properties. It is important to estimate the parameters of the link function, rather than assume a known value. To that end, we present a generalized importance sampling (GIS) estimator based on multiple Markov chains for empirical Bayes analysis of SGLMMs. The GIS estimator, although more efficient than the simple importance sampling, can be highly variable when used to estimate the parameters of certain link functions. Using suitable reparameterizations of the Monte Carlo samples, we propose modified GIS estimators that do not suffer from high variability. We use Laplace approximation for choosing the multiple importance densities in the GIS estimator. Finally, we develop a methodology for selecting models with appropriate link function family, which extends to choosing a spatial correlation function as well. We present an ensemble prediction of the mean response by appropriately weighting the estimates from different models. The proposed methodology is illustrated using simulated and real data examples.
\end{abstract}

Keywords: Geostatistics; Laplace approximation; Markov chain Monte Carlo; multiple importance sampling; model selection; reverse logistic regression.

\section{Introduction}

Spatial generalized linear mixed models (SGLMMs), introduced by Diggle et al. (1998), are often used for analyzing non-Gaussian spatial data that are observed in a continuous region (see e.g. Zhang, 2002; Christensen and Waagepetersen, 2002; Diggle et al., 2003; Christensen, 2004). SGLMMs are generalized linear mixed models where the random effects consist of a spatial process. Conditional on the spatial process, the response variables are assumed to follow a distribution which only depends on the site-specific conditional means. A link function relates the means of the

Address for correspondence: Evangelos Evangelou, Department of Mathematical Sciences, University of Bath, Bath, BA2 7AY, UK. email: ee224@bath.ac.uk 
response variable to the underlying spatial process. For the binomial response variable, a logit or probit link is often assumed, while for the Poisson distribution, a logarithmic link is used. It has been recently shown that the use of a flexible parametric family of link functions (instead of a known fixed link) may produce better inference and prediction (Christensen, 2004; Roy et al., 2016).

Parametric links have been discussed in the literature of generalized linear models (GLMs). For the binomial GLM, for modeling dose-response curves, Prentice (1976) introduces a two-parameter link function given by the quantile of the logarithm of an $F$-distributed random variable, also called the type IV generalized logistic distribution (Johnson et al., 1995). This link function includes the logit and probit links as special cases. Liu (2004), Koenker and Yoon (2009), and Rov (2014) discuss the link function defined by the quantile of the Student's $t$ distribution, the so-called robit link, which approximates the logit and probit links but provides robust inference in the presence of outlying observations. Wang and Dev (2010) use the extreme-value quantile link function which is non-symmetric and can therefore be used when the rate of change in the success probability approaches 0 at a different rate than it approaches 1 . Other authors discussing parametric links for binary data include Aranda-Ordaz (1981); Guerrero and Johnson (1982); Stukel (1988); Nagler (1994); Chen et al. (1999) and Bazán et al. (2006). For Poisson data, Basu and Rathouz (2005) use a Box-Cox link function.

The added flexibility of parametric links introduces the complexity of having to estimate the parameters of the link function. In general, for SGLMMs, the likelihood function can be written only as a multi-dimensional integral and does not have a closed form expression. One way to approximate the intractable likelihood in SGLMMs is by importance sampling (Christensen, 2004). Samples are generated from an importance sampling distribution which are then used for approximating the likelihood by calculating Monte Carlo (MC) averages. The accuracy of the approximation depends on the choice of the importance sampling distribution which can be difficult to elicit if one has to estimate the likelihood for a wide range of parameter values. Generalized importance sampling (GIS) is an efficient importance sampling methodology based on multiple proposal (importance) densities for estimating the ratios of marginal likelihoods for SGLMMs. These ratios of marginal likelihoods are called Bayes factors (BFs). If the marginal likelihood in the denominator (of BFs) is fixed at a parameter value, while the parameter in the numerator is allowed to vary, then maximization of the BFs is equivalent to maximization of the marginal likelihoods resulting in the empirical Bayes (EB) estimate. Rov et al. (2015, 2016) used this idea to estimate not only the link parameter but other parameters as well, such as the spatial range and relative nugget. One benefit of using the EB methodology over a fully Bayesian approach is that it avoids having to specify a prior for these parameters as prior elicitation for these parameters is often difficult, and improper priors on these parameters generally lead to improper posteriors (Berger et al., 2001; Christensen and Waagepetersen, 2002). Also in case of a fully Bayesian analysis, the Markov chain Monte Carlo (MCMC) algorithms may suffer from slow mixing (Christensen, 2004; Roy, 2014).

In this paper we use an EB methodology, implemented by an efficient GIS based on reparameterizations of the MC samples, to fit SGLMMs with parametric links. The contributions of the paper are in four areas:

- Link functions suitable for spatial data analysis. Despite the abundance of parametric link functions in the literature, not every link function is suitable for spatial data analysis, where the link function relates the mean response to the latent spatial field. Because the latent spatial field is assumed to be a Gaussian process, it is required that the link function maps onto the whole real line. Otherwise, this creates an inconsistency in the model because not every possible value of the Gaussian process can correspond to a mean value in the distribution of the observations. 
Some popular link functions discussed in the literature, including the Box-Cox link, do not satisfy this requirement. This fact was noted by Christensen (2004) in the case of the Poisson Box-Cox model. In this paper we provide modifications of these links, by smoothing transitions to their limits, which inherit their flexibility, but also are consistent with the SGLMM. These link functions have not been proposed before in the literature, even for traditional GLMs.

- Improved GIS estimators via reparameterization and control variates. When approximating integrals numerically, a suitable change-of-variables can improve numerical stability. For importance sampling integration, this corresponds to transforming the MC samples. It has been shown that reparameterizations can drastically improve mixing of Gibbs samplers (see e.g. Simpson et al., 2017; Rov, 2014; van Dyk and Meng, 2001; Liu and Wu, 1999). We show in this paper how the GIS estimator without transformation of Roy et al. (2016) can produce biased estimates. We then discuss how to choose suitable transformations to produce better estimators. Thus we derive modified GIS estimators based on transformed (reparameterized) samples. Because of the additional computational cost of transforming the MC samples, some transformations can be slow. In this case, we show how a different, suitable transformation can produce accurate results in less computational time. We also use the proposed transformations to improve the performance of Geyer's (1994) reverse logistic regression estimator. Although Christensen (2004) suggested the use of the mean transformation for the simple importance sampling estimator, this paper is the first to present generalized importance sampling estimators based on general transformations.

Another approach for reducing the variability of IS estimates is the use of control variates (Owen and Zhou, 2000). Doss (2010) used control variates to reduce the variability of BF estimates for multiple IS estimators. We show in this paper how the approach of Doss (2010) can be applied to the reparameterized GIS estimators we propose.

- Selection of proposal distributions using Laplace approximation. The performance of any IS estimator, including GIS, crucially depends on the proposal (importance) distributions. In the literature, there is no systematic method available for selecting these proposal distributions, although it has been generally deemed as difficult (Buta and Doss, 2011). Use of good importance densities is particularly important for spatial models due to potential multimodality of the likelihoods (Mardia and Watkins, 1989). Choosing representative importance sampling distributions can be very difficult if there are too many parameters to estimate. In this paper we use Laplace approximation to integrate out the latent spatial field and thus derive an approximation to the marginal likelihood of the observed data. This approximation is used to choose "good", representative importance sampling distributions.

- Model selection. A typical problem faced by practitioners is the choice among different spatial correlation families and the choice of the link function. An established measure of model comparison and weighting is AIC. However, calculation of AIC is not straightforward for SGLMMs as the likelihood is intractable. This paper is the first to address the problem of spatial model selection using GIS. We demonstrate how the GIS estimator developed in this paper can be used to approximate the AIC by evaluating the Bayes factors between the candidate models. The approximated AIC can be used for model selection and weighting in the spirit of Buckland et al. (1997), thus providing ensemble estimation and prediction methods.

The remainder of the paper is organized as follows. In Section 2 we discuss the SGLMM, and present some suitable link functions for binomial and Poisson/gamma models. In Section 3 
we develop the estimation methodology and a method for selection of importance densities. This section also contains a measure of comparison between models with different link and correlation function families. In Section 4 we use simulation studies to demonstrate the issues with importance sampling and how our modified methods based on transformation can address these. We also demonstrate the performance of the proposed model selection criterion via a different simulation study. The methods discussed in this paper are applied to two real-data examples in Section 5 , Finally, Section 6 presents the conclusions of this paper. Some technical derivations are relegated to Appendix A. A summary of the steps involved in the proposed computational and inferential procedure is presented in Appendix B. Finally, Appendix C contains further details about the examples.

\section{Spatial generalized linear mixed models}

Let $\{Z(s), s \in \mathbb{S}\}$ be a Gaussian random field with mean function $E(Z(s))=\sum_{j=1}^{p} x_{j}(s) \beta_{j}$, where $\beta=\left(\beta_{1}, \ldots, \beta_{p}\right)^{\prime} \in \mathcal{R}^{p}$ are the unknown regression parameters, $\mathbf{x}(s)=\left(x_{1}(s), \ldots, x_{p}(s)\right)$ are the known location dependent covariates, and the covariance function $\operatorname{Cov}\left(Z(s), Z\left(s^{\prime}\right)\right)=\sigma^{2} \rho_{\theta}\left(s, s^{\prime}\right)+$ $\tau^{2} I_{\left\{s=s^{\prime}\right\}}$. Here $\rho_{\theta}\left(s, s^{\prime}\right)$ is the spatial correlation function which models the dependence between distinct locations. In this paper we assume a stationary and isotropic correlation, i.e. $\rho_{\theta}\left(s, s^{\prime}\right)=$ $\rho_{\theta}\left(\left\|s-s^{\prime}\right\|\right)$, where $\left\|s-s^{\prime}\right\|$ denotes the Euclidean distance between $s$ and $s^{\prime}$. Some examples of correlation functions are the Matérn, the exponential-power, and the spherical parametric families (Diggle et al., 2003). These functions depend on parameters $\theta$. In the case of the spherical family, there is only one parameter, the spatial range $\phi$, i.e. $\theta=\{\phi\}$, but in the case of the Matérn and exponential-power families, there is respectively an additional smoothness or power parameter $\kappa$, i.e. $\theta=\{\phi, \kappa\}$. The parameter $\sigma^{2}$ is called the partial sill, and $\tau^{2}$ is called the nugget effect. The nugget effect can be interpreted as micro-scale variation, measurement error, or a combination of both. It is convenient to let $\omega=\tau^{2} / \sigma^{2}$ and write the covariance as $\operatorname{Cov}\left(Z(s), Z\left(s^{\prime}\right)\right)=\sigma^{2}\left[\rho_{\theta}\left(s, s^{\prime}\right)+\omega I_{\left\{s=s^{\prime}\right\}}\right]$.

Conditional on the realized value of the Gaussian random field, $\{z(s), s \in \mathbb{S}\}$, the response/observation process $\{Y(s), s \in \mathbb{S}\}$ is assumed to consist of independent random variables, and for each $s \in \mathbb{S}$ the distribution of $Y(s) \mid z(s)$ has conditional mean

$$
\mathrm{E}(Y(s) \mid z(s))=t(s) \mu(s)
$$

where $t(s)$ is a known function and $\mu(s)$ is related to $z(s)$ through a link function $h_{\nu}$ such that

$$
h_{\nu}(\mu(s))=z(s) .
$$

The Gaussian random field is unobserved while the response process is observed at a finite set of locations $s_{1}, \ldots, s_{n} \in \mathbb{S}$. We write $y_{i}=y\left(s_{i}\right), \mu_{i}=\mu\left(s_{i}\right)$ and so on.

The link function $h_{\nu}$ is assumed to belong to a parametric family depending on parameters $\nu$. The conditional model for the observation process depends on $z(s)$ only through its relationship with $\mu(s)$ and can be written as

$$
p[y(s) \mid z(s) ; \nu]=p\left[y(s) \mid \mu(s)=f_{\nu}(z(s))\right],
$$

where we use $p[\cdot]$ to denote the pmf/pdf of the enclosed expression. We also use $f_{\nu}(\cdot)=h_{\nu}^{-1}(\cdot)$ to denote the inverse of the link function.

We now present two examples of SGLMMs appropriate for binary and count data respectively. Roy et al. (2016) consider the following robust SGLMM for analyzing spatial binomial data. For any $s_{1}, \ldots, s_{n} \in \mathbb{S}$, conditional on $\{z(s)\}$, the response variables $Y_{1}, \ldots, Y_{n}$ are assumed to follow 
$Y_{i} \mid z_{i} \stackrel{\text { ind }}{\sim} \operatorname{Binomial}\left(t_{i}, \mu_{i}\right)$ with $\mu_{i}=G_{\nu}\left(z_{i}\right)$, where $G_{\nu}(\cdot)$ is the cumulative distribution function (cdf) of the standard Student's $t$ distribution with degrees of freedom $\nu$ and $t_{i}$ is a known constant (number of trials at the location $s_{i}$ ) for $i=1, \ldots, n$. This model is called the spatial robit model because it is more robust to outlying observations compared to the standard logistic and probit models.

Our second example is used to analyze spatial count data, where $Y_{i} \mid z_{i} \stackrel{\text { ind }}{\sim} \operatorname{Poisson}\left(t_{i} \mu_{i}\right)$, with $\mu_{i}=h_{\nu}^{-1}\left(z_{i}\right)$. Here $t_{i}$ may represent the length of the recording period over which $y_{i}$ is observed, or the area within which $y_{i}$ is counted. Christensen (2004) considers the Box-Cox family of link functions given by

$$
h_{\nu}\left(\mu_{i}\right)= \begin{cases}\left(\mu_{i}^{\nu}-1\right) / \nu, & \text { if } \nu \neq 0, \\ \log \left(\mu_{i}\right), & \text { if } \nu=0 .\end{cases}
$$

So the commonly used log link function, $h\left(\mu_{i}\right)=\log \left(\mu_{i}\right)$ is a special case of the above Box-Cox family of link functions. For analyzing a data set of radionuclide concentrations on Rongelap island, Christensen (2004) provides evidence that the log-link, as used by Diggle et al. (1998), may not be a good choice and uses the above Box-Cox family of link functions.

A problem with the Box-Cox link is that it is inconsistent with the SGLM model for $\nu \neq 0$ because it imposes the restrictions $z_{i}>-1 / \nu$ and $z_{i}<-1 / \nu$ if $\nu>0$ or $\nu<0$ respectively, which contradicts the Gaussian assumption for $z_{i}$. To avoid this issue, Christensen (2004) extended the model to allow for $z_{i} \in \mathcal{R}$ such that $z_{i} \in(-\infty,-1 / \nu] \Leftrightarrow \mu_{i}=0$ if $\nu>0$, and $z_{i} \in[-1 / \nu, \infty) \Leftrightarrow$ $\mu_{i}=0$ if $\nu<0$, and $\mu_{i}=0 \Rightarrow y_{i}=0$ with probability 1 . However in this case the link function is not invertible.

\subsection{Parametric link functions}

We now discuss some desirable properties of link functions. To facilitate inference, we require the function to be monotone and differentiable. In order to be consistent with the SGLMM, we require that the function maps the range of values for the mean (of the observation process) onto the real line. This property is not satisfied, for example by the Box-Cox link used in Christensen (2004) when $\nu \neq 0$. We present below some suggestions for parametric links for different models.

\subsubsection{Binomial response variables}

For binomial response variables, the mean, $f_{\nu}(z)$, lies between 0 and 1 . It is helpful to think of the inverse link function as having the form $f_{\nu}(z)=F_{\nu}(z)$ where $F_{\nu}$ is the cdf of a real-valued continuous random variable with support being the whole real line. The popular logistic and probit models are derived by letting $F_{\nu}$ be the cdf of the logistic and standard normal distributions respectively, while the robit link of Liu (2004) corresponds to the cdf of the standard Student's $t_{\nu}$ distribution.

Roy et al. (2016) demonstrate the advantages of using a parametric link function for robust spatial inference under model misspecification, or in the presence of outlying observations. In the latter case, the robit link function with low degrees of freedom would be more appropriate choice. Similar behavior can be achieved by using a computationally efficient approximation to the $t_{\nu}$ cdf due to Wallace (1959), that is,

$$
F_{\nu}(z)=\Phi(\zeta), \zeta=\operatorname{sign}(z) \frac{8 \nu+1}{8 \nu+3} \sqrt{\nu \log \left(1+z^{2} / \nu\right)},
$$

where $\Phi(\cdot)$ denotes the cdf of the standard normal distribution. 
In other situations, one may want to use a non-symmetric cdf, if e.g. the rates at which the success probability approaches 0 and 1 are different. The generalized extreme value (GEV) link was proposed by Wang et al. (2010) and can be used for this purpose. This link corresponds to

$$
F_{\nu}(z)= \begin{cases}\exp \left\{-\max (0,1+\nu z)^{-1 / \nu}\right\}, & \text { if } \nu \neq 0, \\ \exp \{-\exp (-z)\}, & \text { if } \nu=0,\end{cases}
$$

which puts restrictions on $z$ as $z>-1 / \nu$ if $\nu>0$ and $z<-1 / \nu$ if $\nu<0$. A link that behaves similarly as (4) but maps onto the real line can be obtained by letting $f_{\nu}(z)=F_{\nu}(z)$ where

$$
F_{\nu}(z)= \begin{cases}\exp \left\{-(1+|\nu||z|)^{-\operatorname{sign}(z) /|\nu|}\right\}, & \text { if } \nu \neq 0, \\ \exp \{-\exp (-z)\}, & \text { if } \nu=0 .\end{cases}
$$

We will refer to the link corresponding to (5) as the modified GEV link. Since this link function depends only on $|\nu|$, either $\nu \in(-\infty, 0]$, or $\nu \in[0, \infty)$ is assumed.

One advantage of the standard GEV link is that it allows for positive as well as negative skewness while the modified GEV link only allows for positive skewness. This means that the modified GEV only considers the case where the probability of success approaches 1 faster than it approaches 0 , and may result in poor fit for some data. However, for every $\operatorname{cdf} F_{\nu}(z), F_{\nu}^{*}(z)=1-F_{\nu}(-z)$ is a also a cdf, and if $F_{\nu}(z)$ generates a positively skewed link, then $F_{\nu}^{*}(z)$ generates a negatively skewed link. This is equivalent to interpreting successes as failures and vice versa. When $F_{\nu}(z)$ is the cdf in (5) then $F_{\nu}^{*}(z)$ corresponds to the negatively-skewed modified GEV link, which includes the popular complementary log-log link as a special case.

\subsubsection{Poisson and gamma response variables}

For Poisson and gamma models the mean response can take any positive real value. A general family of inverse link functions can be derived by

$$
f_{\nu}(z)=-\log F_{\nu}(-z),
$$

where $F_{\nu}(z)$ is a cdf as in the binomial case. For instance the choice $f_{\nu}(z)=-\log F_{-\nu}(-z)$ where $F_{\nu}$ is the GEV cdf in (41) produces the Box-Cox link (2) with the logarithmic link as a special case. Thus a modified Box-Cox link can be derived by using the modified GEV cdf (given in (5)) as

$$
h_{\nu}(\mu)= \begin{cases}\frac{\mu^{\nu}-1}{\nu} & \text { if } \nu>0 \text { and } \mu \geq 1 \\ \frac{1-\mu^{-\nu}}{\nu} & \text { if } \nu>0 \text { and } \mu<1 \\ \log \mu & \text { if } \nu=0\end{cases}
$$

\section{Empirical Bayes estimation of SGLMMs}

Suppose that the data $\mathbf{y}=\left(y_{1}, \ldots, y_{n}\right)$ consist of a single realization of the process $\{Y(s), s \in \mathbb{S}\}$ mentioned in Section 2 at known sampling locations $s_{1}, \ldots, s_{n} \in \mathbb{S}$. Let us divide all unknown parameters into two categories $\psi \equiv\left(\beta, \sigma^{2}\right)$ and $\xi \equiv(\nu, \theta)$ depending on whether a conjugate prior for those parameters given $\mathbf{z}$ exists or not respectively. One of the reasons for this split is that it is straightforward to sample from the full conditionals of the parameters in $\psi$, as these are standard distributions, but not so if we had assumed a prior for $\xi$. The likelihood function of SGLMM is not available in closed form, but only as a high dimensional integral, that is,

$$
L_{\xi}(\psi \mid \mathbf{y}) \equiv L(\psi, \xi \mid \mathbf{y})=\int_{\mathcal{R}^{n}} p[\mathbf{y}, \mathbf{z} \mid \psi, \xi] \mathrm{d} \mathbf{z}=\int_{\mathcal{R}^{n}} p[\mathbf{y} \mid \mathbf{z}, \nu] p[\mathbf{z} \mid \psi, \xi] \mathrm{d} \mathbf{z}
$$


where $\mathbf{z}=\left(z_{1}, \ldots, z_{n}\right), z_{i} \equiv z\left(s_{i}\right), p[\mathbf{y} \mid \mathbf{z}, \nu]=\prod_{i=1}^{n} p\left[y_{i} \mid z_{i}, \nu\right]$ with $p\left[y_{i} \mid z_{i}, \nu\right]=p\left[y_{i} \mid \mu_{i}=f_{\nu}\left(z_{i}\right)\right]$ being the conditional density of $y_{i} \mid z_{i}$, and $p[\mathbf{z} \mid \psi, \xi]$ is the multivariate Gaussian density for $\mathbf{z}$ with mean vector $X \beta$ and covariance matrix involving the parameters $\sigma^{2}$ and $\theta$, and $X$ is the known $n \times p$ matrix defined by $X_{i j}=x_{j}\left(s_{i}\right)$.

Note that the Gaussian prior for $\beta$ (conditional on $\sigma^{2}$ ) and scaled inverse chi-square prior for $\sigma^{2}$ are conjugate priors for $\psi=\left(\beta, \sigma^{2}\right)$ for the joint density $p[\mathbf{y}, \mathbf{z} \mid \psi, \xi]$. Let $\pi(\psi)$ be the prior on $\psi$ obtained from assuming $\beta \mid \sigma^{2} \sim N\left(m_{b}, \sigma^{2} V_{b}\right)$, and $\sigma^{2} \sim \chi_{S c I}^{2}\left(n_{\sigma}, a_{\sigma}\right)$ where the hyperparameters $m_{b}, V_{b}, a_{\sigma}, n_{\sigma}$ are assumed known.

Consider the augmented joint density $p[\mathbf{y}, \mathbf{z} \mid \psi, \xi]$ and the corresponding so-called complete posterior density

$$
\pi_{\xi}(\psi, \mathbf{z} \mid \mathbf{y})=\frac{p[\mathbf{y}, \mathbf{z} \mid \psi, \xi] \pi(\psi)}{m_{\xi}(\mathbf{y})}
$$

where

$$
m_{\xi}(\mathbf{y})=\int_{\mathcal{R}^{p} \times \mathcal{R}_{+}} \int_{\mathcal{R}^{n}} p[\mathbf{y}, \mathbf{z} \mid \psi, \xi] \pi(\psi) \mathrm{d} \mathbf{z} \mathrm{d} \psi=\int_{\mathcal{R}^{p} \times \mathcal{R}_{+}} L_{\xi}(\psi \mid \mathbf{y}) \pi(\psi) \mathrm{d} \psi
$$

is the normalizing constant (also known as the marginal density). The empirical Bayes (EB) approach to inference is to estimate $\xi$ by maximizing this marginal density. Suppose $\hat{\xi}$ is the maximizer, i.e, $\hat{\xi}=\operatorname{argmax} m_{\xi}(\mathbf{y})$. Then, the posterior density $\pi_{\hat{\xi}}(\psi, \mathbf{z} \mid \mathbf{y})$ of $(\psi, \mathbf{z})$, conditioned on the observed data $\mathbf{y}$ and $\xi=\hat{\xi}$ is used to infer about $(\psi, \mathbf{z})$. Typically, for fixed $\xi$, one would sample iteratively from the full conditionals $\pi_{\xi}(\psi \mid \mathbf{z}, \mathbf{y})$ and $\pi_{\xi}(\mathbf{z} \mid \psi, \mathbf{y})$ to run a Gibbs sampler. Since we use conjugate priors for $\psi$, sampling from the former is straightforward, while for the latter a Metropolis-Hastings algorithm is used as in Diggle et al. (1998).

Note that for any arbitrary fixed $\xi_{1}, \hat{\xi}$ is equal to $\hat{\xi}=\operatorname{argmax} B_{\xi, \xi_{1}}$ where $B_{\xi, \xi_{1}}=m_{\xi}(\mathbf{y}) / m_{\xi_{1}}(\mathbf{y})$ is the $\mathrm{BF}$ for the model indexed by $\xi$ relative to the model indexed by $\xi_{1}$. The reason for considering the latter is that it is often much easier to compute the ratio $B_{\xi, \xi_{1}}$ instead of the marginal likelihood $m_{\xi}(\mathbf{y})$ directly. (Note that in order to find the maximizer of $B_{\xi, \xi_{1}}$ we may need to estimate $B_{\xi, \xi_{1}}$ for many values of $\xi$.) For example if $\left\{\psi^{(i)}, \mathbf{z}^{(i)}\right\}_{i=1}^{N}$ is a positive Harris Markov chain with stationary density $\pi_{\xi_{1}}(\psi, \mathbf{z} \mid \mathbf{y})$, then a consistent estimator of $B_{\xi, \xi_{1}}$ is given by

$$
\frac{1}{N} \sum_{i=1}^{N} \frac{p\left[\mathbf{y}, \mathbf{z}^{(i)} \mid \psi^{(i)}, \xi\right]}{p\left[\mathbf{y}, \mathbf{z}^{(i)} \mid \psi^{(i)}, \xi_{1}\right]} \stackrel{\text { a.s. }}{\longrightarrow} \int_{\mathcal{R}^{n}} \int_{\mathcal{R}^{p} \times \mathcal{R}_{+}} \frac{p[\mathbf{y}, \mathbf{z} \mid \psi, \xi]}{p\left[\mathbf{y}, \mathbf{z} \mid \psi, \xi_{1}\right]} \pi_{\xi_{1}}(\psi, \mathbf{z} \mid \mathbf{y}) \mathrm{d} \psi d \mathbf{z}=\frac{m_{\xi}(\mathbf{y})}{m_{\xi_{1}}(\mathbf{y})},
$$

as $N \rightarrow \infty$, where $p[\mathbf{y}, \mathbf{z} \mid \psi, \xi]$ is the joint density given in ([6]). The simple importance sampling (IS) estimator (9) is often unstable as some of the terms (ratios of densities) take very large values especially when $\xi$ is not "close" to $\xi_{1}$ (Gever, 1996; Christensen, 2004; Doss, 2010).

We now describe the GIS method for estimating $\hat{\xi}$. A more efficient method for estimating $B_{\xi, \xi}$ for a wide range of values for $\xi$ was proposed initially by Gever (1994) (see also Gever and Thompson, 1992) and subsequently used by Doss (2010) and Roy et al. (2016) among others. The idea is to choose a skeleton set $\Xi=\left\{\xi_{1}, \ldots, \xi_{k}\right\}$ with multiple $\xi$ values and generate a Markov chain $\left\{\psi^{(j ; l)}, \mathbf{z}^{(j ; l)}\right\}_{l=1}^{N_{j}}$ with stationary density $\pi_{\xi_{j}}(\psi, \mathbf{z} \mid \mathbf{y})$ for each $j=1, \ldots, k$ and use the following generalized IS (GIS) estimator

$$
\hat{B}_{\xi, \xi_{1}}(\hat{\mathbf{r}})=\sum_{j=1}^{k} \sum_{l=1}^{N_{j}} \frac{p\left[\mathbf{y}, \mathbf{z}^{(j ; l)} \mid \psi^{(j ; l)}, \xi\right]}{\sum_{i=1}^{k} N_{i} p\left[\mathbf{y}, \mathbf{z}^{(j ; l)} \mid \psi^{(j ; l)}, \xi_{i}\right] / \hat{r}_{i}},
$$

where $\hat{\mathbf{r}}=\left(\hat{r}_{1}, \hat{r}_{2}, \ldots, \hat{r}_{k}\right)$ is the "reverse logistic regression" (RL) estimator (Geyer, 1994) of $\mathbf{r}=$ $\left(r_{1}, r_{2}, \ldots, r_{k}\right)$ with $r_{i} \equiv m_{\xi_{i}}(\mathbf{y}) / m_{\xi_{1}}(\mathbf{y})$ for $i=2, \ldots k$ and $\hat{r}_{1}=1=r_{1}$. This leads to a numerically stable IS estimator with smaller variance than (9). 
In order to describe Geyer's (1994) RL estimation of r, define

$$
\delta_{j}=-\log r_{j}+\log \frac{N_{j}}{N} \quad \text { for } j=1, \ldots, k,
$$

where $N=\sum_{j=1}^{k} N_{j}$. The RL estimator of $\delta=\left(\delta_{1}, \ldots, \delta_{k}\right)$ (and hence of $\mathbf{r}$ ) is obtained by maximizing the log quasi likelihood function

$$
\sum_{j=1}^{k} \sum_{l=1}^{N_{j}} \log \tilde{p}_{j}\left(\psi^{(j ; l)}, \mathbf{z}^{(j ; l)} ; \delta\right) \quad \text { with the constraint } \sum_{j=1}^{k} \delta_{j}=0,
$$

where

$$
\tilde{p}_{j}(\psi, \mathbf{z} ; \delta)=\frac{p\left[\mathbf{y}, \mathbf{z} \mid \psi, \xi_{j}\right] e^{\delta_{j}}}{\sum_{t=1}^{k} p\left[\mathbf{y}, \mathbf{z} \mid \psi, \xi_{t}\right] e^{\delta_{t}}} .
$$

Note that, $\tilde{p}_{j}(\psi, \mathbf{z} ; \delta)$ is the probability that $(\psi, \mathbf{z})$ came from the $j$ th density $\pi_{\xi_{j}}(\psi, \mathbf{z} \mid \mathbf{y})$ given that it belongs to the pooled sample $\left\{\psi^{(j ; l)}, \mathbf{z}^{(j ; l)}, l=1, \ldots, N_{j}, j=1, \ldots, k\right\}$. The reason for the constraint in (12) is because the $\delta_{i}$ 's are only identifiable up to a constant, i.e., adding a fixed constant to (11) does not change (13). This unidentifiability is not an issue for us because we only need to estimate $k-1$ ratios $r_{j}, j=2, \ldots, k$.

Doss (2010) proposed a two stage scheme for using the GIS estimator (10). In the 1st stage

based on samples $\left\{\psi^{(j ; l)}, \mathbf{z}^{(j ; l)}\right\}_{l=1}^{\tilde{N}_{j}}$ from $\pi_{\xi_{j}}(\psi, \mathbf{z} \mid \mathbf{y}), j=1, \ldots, k, \mathbf{r}$ (the ratios of marginal likelihoods at $k$ skeleton points) is estimated by the RL method. Then independent of stage I, new samples $\left\{\psi^{(j ; l)}, \mathbf{z}^{(j ; l)}\right\}_{l=1}^{N_{j}}$ are obtained from $\pi_{\xi_{j}}(\psi, \mathbf{z} \mid \mathbf{y}), j=1, \ldots, k$ to estimate $m_{\xi}(\mathbf{y}) / m_{\xi_{1}}(\mathbf{y})$ for all $\xi$ using (10). Rov et al. (2018) provide standard error estimates of $\hat{\mathbf{r}}$ and $\hat{B}_{\xi, \xi_{1}}(\hat{\mathbf{r}})$ that can be used for deciding the appropriate sample sizes $\tilde{N}_{j}$ 's and $N_{j}$ 's. This two-stage GIS estimator was used in Roy et al. (2016) for EB estimation in the binomial SGLMM with robit link. More details about this procedure are given in Appendix B. However, as with the naive IS method, the variability of (13) can be high if the importance densities do not sufficiently "overlap". This issue is overcome using reparameterizations.

\subsection{Reparameterized generalized importance sampling estimators}

It turns out that, under certain circumstances, the GIS estimator (10) can be unreliable although it is more efficient than the naive IS estimator (9). The reason is that the functions $\mathbf{z} \mapsto p[\mathbf{y} \mid \boldsymbol{\mu}=$ $\left.f_{\nu}(\mathbf{z})\right]$ and $\mathbf{z} \mapsto p\left[\mathbf{y} \mid \boldsymbol{\mu}=f_{\nu^{\prime}}(\mathbf{z})\right]$ can be very different when $\nu \neq \nu^{\prime}$ (see e.g. Christensen, 2004). Consequently, the Monte Carlo sample will be separable (see Gever, 1994) if the points in the skeleton set are not sufficiently close. For example, suppose $\mathbf{z}$ is a sample (generated by a Metropolis-Hastings algorithm) from the (z marginal) posterior density (7) corresponding to the Poisson SGLMM with the Box-Cox link with exponent $\nu=1$. Thus, most likely, the sampled $\mathbf{z}$ assigns appreciable mass to the probability $p[\mathbf{y} \mid \boldsymbol{\mu}=\mathbf{z}+1]$. The RL estimator and (10) require that we evaluate $p\left[\mathbf{y} \mid \boldsymbol{\mu}=f_{\nu^{\prime}}(\mathbf{z})\right]$ at all other $\nu^{\prime}$ in the skeleton set $\Xi$. If $\nu^{\prime}=0$ this becomes $p[\mathbf{y} \mid \boldsymbol{\mu}=\exp (\mathbf{z})]$ so the mean of the Poisson distribution changes drastically even for moderate values of $\mathbf{z}$, and, in effect, the probability corresponding to $\nu^{\prime}$ can be numerically indistinguishable from 0 .

To avoid this issue, we consider reparameterizations of the integral in (6) . To that end, write the likelihood in (6) as an integral with respect to $\boldsymbol{\mu}$ instead of $\mathbf{z}$. Consider the transformation $h_{\nu}^{-1}: \mathbf{z} \mapsto \boldsymbol{\mu}$ which is valid only when the link function is invertible over the whole real line. The Jacobian of the transformation is $\tilde{J}_{\nu}(\boldsymbol{\mu})=\prod_{i=1}^{n} h_{\nu}^{\prime}\left(\mu_{i}\right)$. As in (7), the corresponding complete 
posterior density of $(\psi, \boldsymbol{\mu})$ is

$$
\pi_{\xi}(\psi, \boldsymbol{\mu} \mid \mathbf{y})=\frac{p[\mathbf{y}, \boldsymbol{\mu} \mid \psi, \xi] \pi(\psi)}{m_{\xi}(\mathbf{y})}
$$

based on the augmented joint density $p[\mathbf{y}, \boldsymbol{\mu} \mid \psi, \xi]=p[\mathbf{y} \mid \boldsymbol{\mu}] p\left[\mathbf{z}=h_{\nu}(\boldsymbol{\mu}) \mid \psi, \xi\right] \tilde{J}_{\nu}(\boldsymbol{\mu})$. Note that if we have a Markov chain $\left\{\psi^{(i)}, \mathbf{z}^{(i)}\right\}_{i \geq 0}$ with stationary density $\pi_{\xi}(\psi, \mathbf{z} \mid \mathbf{y})$ then $\left\{\psi^{(i)}, \boldsymbol{\mu}^{(i)}=f_{\nu}\left(\mathbf{z}^{(i)}\right)\right\}_{i \geq 0}$ is a Markov chain with stationary density $\pi_{\xi}(\psi, \boldsymbol{\mu} \mid \mathbf{y})$ given in (14). The advantage of using the latter is that the estimator (10) now becomes

$$
\tilde{B}_{\xi, \xi_{1}}(\tilde{\mathbf{r}})=\sum_{j=1}^{k} \sum_{l=1}^{N_{j}} \frac{p\left[\mathbf{z}=h_{\nu}\left(\boldsymbol{\mu}^{(j ; l)}\right) \mid \psi^{(j ; l)}, \xi\right] \tilde{J}_{\nu}\left(\boldsymbol{\mu}^{(j ; l)}\right)}{\sum_{i=1}^{k} N_{i} p\left[\mathbf{z}=h_{\nu_{i}}\left(\boldsymbol{\mu}^{(j ; l)}\right) \mid \psi^{(j ; l)}, \xi_{i}\right] \tilde{J}_{\nu_{i}}\left(\boldsymbol{\mu}^{(j ; l)}\right) / \tilde{r}_{i}},
$$

which, unlike (10), does not involve the conditional pmf of $\mathbf{y}, p\left[\mathbf{y} \mid \boldsymbol{\mu}=f_{\nu}(\mathbf{z})\right]$. In (15), we use $\tilde{r}_{i}$ to denote RL estimator of $r_{i}$ based on the transformed $\mathrm{MC}$ samples, i.e., by using $p[\mathbf{z}=$ $\left.h_{\nu_{j}}(\boldsymbol{\mu}) \mid \psi, \xi_{j}\right] \tilde{J}_{\nu_{j}}(\boldsymbol{\mu})$ instead of $p\left[\mathbf{y}, \mathbf{z} \mid \psi, \xi_{j}\right]$, for $j=1, \ldots, k$ in (13).

The use of (15) presents two new challenges. First, it is valid only when the link function maps onto the whole real line, therefore it cannot, in general, be used with the Box-Cox link (2) or the GEV link (4). Secondly, computing $h_{\nu}(\boldsymbol{\mu})$ can be slow, which can add significant computing time when evaluated over many different values of $\boldsymbol{\mu}$. Such is the case for the robit link when $\nu<1$ (see Koenker (2006) and Remark 5 in Cran et al. (1977)).

More generally, we can use any transformation $g_{\nu}^{-1}: \mathbf{z} \mapsto \mathbf{w}$, not necessarily the link. Here $\mathbf{w}=\left(w_{1}, \ldots, w_{n}\right)$. If chosen appropriately such that $w_{i} \approx \mu_{i}$, it can alleviate the separability problem. For example, if Box-Cox or GEV link is used for analyzing data, then the modified versions of the Box-Cox and GEV links introduced in sections 2.1.1 and 2.1.2 can be used for constructing this transformation. In the case of the robit link, it can be the Wallace transformation (3) which is computationally faster. Define the complete posterior density of $(\psi, \mathbf{w})$,

$$
\pi_{\xi}(\psi, \mathbf{w} \mid \mathbf{y})=\frac{p\left[\mathbf{y} \mid \boldsymbol{\mu}=f_{\nu}\left(g_{\nu}(\mathbf{w})\right)\right] p\left[\mathbf{z}=g_{\nu}(\mathbf{w}) \mid \psi, \xi\right] \bar{J}_{\nu}(\mathbf{w}) \pi(\psi)}{m_{\xi}(\mathbf{y})}
$$

where $\bar{J}_{\nu}(\mathbf{w})=\prod_{i=1}^{n} g_{\nu}^{\prime}\left(w_{i}\right)$. The estimator of the Bayes factors in the general case becomes

$$
\bar{B}_{\xi, \xi_{1}}(\overline{\mathbf{r}})=\sum_{j=1}^{k} \sum_{l=1}^{N_{j}} \frac{p\left[\mathbf{y} \mid \boldsymbol{\mu}=f_{\nu}\left(g_{\nu}\left(\mathbf{w}^{(j ; l)}\right)\right)\right] p\left[\mathbf{z}=g_{\nu}\left(\mathbf{w}^{(j ; l)}\right) \mid \psi^{(j ; l)}, \xi\right] \bar{J}_{\nu}\left(\mathbf{w}^{(j ; l)}\right)}{\sum_{i=1}^{k} N_{i} p\left[\mathbf{y} \mid \boldsymbol{\mu}=f_{\nu_{i}}\left(g_{\nu_{i}}\left(\mathbf{w}^{(j ; l)}\right)\right)\right] p\left[\mathbf{z}=g_{\nu_{i}}\left(\mathbf{w}^{(j ; l)}\right) \mid \psi^{(j ; l)}, \xi_{i}\right] \bar{J}_{\nu_{i}}\left(\mathbf{w}^{(j ; l)}\right) / \bar{r}_{i}},
$$

where $\left\{\psi^{(j ; l)}, \mathbf{w}^{(j ; l)}\right\}_{l \geq 0}$ is a Markov chain with stationary density $\pi_{\xi_{j}}(\psi, \mathbf{w} \mid \mathbf{y})$ given in (16), and $\bar{r}_{i}$ is the RL estimator of $r_{i}$ based on the samples $\left\{\psi^{(j ; l)}, \mathbf{w}^{(j ; l)}\right\}_{l>0}$. As before, if we have a Markov chain $\left\{\psi^{(i)}, \mathbf{z}^{(i)}\right\}_{i \geq 0}$ with stationary density $\pi_{\xi}(\psi, \mathbf{z} \mid \mathbf{y})$ then $\left\{\psi^{(i)}, \mathbf{w}^{(i)}=g_{\nu}^{-1}\left(\mathbf{z}^{(i)}\right)\right\}_{i \geq 0}$ is a Markov chain with stationary density $\pi_{\xi}(\psi, \mathbf{w} \mid \mathbf{y})$. Unlike (15), the expression of (17) is not free of the pmf of $\mathbf{y}$, but, as we show through examples in Section 4, (17) can lead to huge gains in computational efficiency over (15) without sacrificing accuracy. Note that the GIS estimators (10) and (15) are special cases of (17) with $g_{\nu}$ being the identity function and $f_{\nu}^{-1}$ respectively. In (17) the RL estimator $\overline{\mathbf{r}}$ is obtained by using $p\left[\mathbf{y} \mid \boldsymbol{\mu}=f_{\nu_{j}}\left(g_{\nu_{j}}(\mathbf{w})\right)\right] p\left[\mathbf{z}=g_{\nu_{j}}(\mathbf{w}) \mid \psi, \xi_{j}\right] \bar{J}_{\nu_{j}}(\mathbf{w})$ instead of $p\left[\mathbf{y}, \mathbf{z} \mid \psi, \xi_{j}\right]$, for $j=1, \ldots, k$ in (13).

Note that the function $g_{\nu}$ can be different for each component of the vector $\mathbf{z}$, so we can apply a different transformation to each component. One example where we want to do that is the case of the Poisson SGLMM with the Box-Cox link where some $y_{i}$ 's are strictly positive, and some other $y_{i}$ 's are equal to zero. As we have already explained, in the latter case the Box-Cox link does not 
map onto the real line so the modified Box-Cox transformation should be used. In case of $y_{i}>0$, we must have $\mu_{i}>0$, but this can fail if $w_{i}$ is simulated conditional on $\nu=\nu_{1}$ and $\mu_{i}=f_{\nu}\left(g_{\nu}\left(w_{i}\right)\right)$ is evaluated at $\nu=\nu_{2}>\nu_{1}$ when $g_{\nu}$ is the modified Box-Cox function. Therefore, we let $g_{\nu}$ be the Box-Cox transformation when $y_{i}>0$ and the modified transformation when $y_{i}=0$.

The estimator (17) can be further improved by the use of control variates (Owen and Zhou, 2000). The use of control variates in the context of GIS estimation was discussed in Doss (2010). Below we use control variates to improve the reparameterized estimator (17). Let $a_{i}=N_{i} / N$,

$$
q_{\xi}(\psi, \mathbf{w})=p\left[\mathbf{y} \mid \boldsymbol{\mu}=f_{\nu}\left(g_{\nu}(\mathbf{w})\right)\right] p\left[\mathbf{z}=g_{\nu}(\mathbf{w}) \mid \psi, \xi\right] \bar{J}_{\nu}(\mathbf{w}),
$$

and define

$$
Y(\psi, \mathbf{w})=\frac{q_{\xi}(\psi, \mathbf{w})}{\sum_{i=1}^{k} a_{i} q_{\xi_{i}}(\psi, \mathbf{w}) / r_{i}},
$$

and for $j=2, \ldots, k$,

$$
X_{j}(\psi, \mathbf{w})=\frac{q_{\xi_{j}}(\psi, \mathbf{w}) / r_{j}-q_{\xi_{1}}(\psi, \mathbf{w})}{\sum_{i=1}^{k} a_{i} q_{\xi_{i}}(\psi, \mathbf{w}) / r_{i}} .
$$

Note that $\operatorname{E} Y(\psi, \mathbf{w})=B_{\xi, \xi_{1}}$ and $\operatorname{E} X_{j}(\psi, \mathbf{w})=0$ where the expectation is taken with respect to the mixture density

$$
\pi_{\operatorname{mix}}(\psi, \mathbf{w} \mid \mathbf{y})=\sum_{i=1}^{k} a_{i} \pi_{\xi_{i}}(\psi, \mathbf{w} \mid \mathbf{y})
$$

Then, for any $\alpha=\left(\alpha_{2}, \ldots, \alpha_{k}\right)$,

$$
\hat{I}_{\alpha}=\frac{1}{N} \sum_{j=1}^{k} \sum_{l=1}^{N_{j}}\left\{Y\left(\psi^{(j ; l)}, \mathbf{w}^{(j ; l)}\right)-\sum_{i=2}^{k} \alpha_{i} X_{i}\left(\psi^{(j ; l)}, \mathbf{w}^{(j ; l)}\right)\right\},
$$

is an unbiased estimator of $B_{\xi, \xi_{1}}$ where the samples are obtained from the density (20). In the case $\alpha=0, \hat{I}_{\alpha}$ reduces to $\bar{B}_{\xi, \xi_{1}}(\mathbf{r})$, but Owen and Zhou (2000) argued that an optimal choice for $\alpha$ is the one that minimizes the variance of (21), in which case $\hat{I}_{\alpha}$ corresponds to the estimate of the intercept term in the least squares regression of $Y\left(\psi^{(j ; l)}, \mathbf{w}^{(j ; l)}\right)$ against $X_{i}\left(\psi^{(j ; l)}, \mathbf{w}^{(j ; l)}\right), i=2, \ldots, k$. In practice, $r_{i}$ is replaced by its reverse logistic regression estimate, $\bar{r}_{i}$, in (18) and (19) before computing $\hat{I}_{\alpha}$.

\subsection{Derivation of skeleton points}

In this section we describe a method of choosing the multiple importance densities corresponding to the mixture distribution used in the GIS estimator (10) and its derivatives based on transformed samples. This boils down to choosing the skeleton set $\Xi$.

Because $B_{\xi, \xi_{1}} \propto m_{\xi}(\mathbf{y})$, the skeleton set is derived by approximating the integral in (8) using integrated, nested Laplace approximations. The approximation can be used to get preliminary estimates of $m_{\xi}(\mathbf{y})$ and thus of $\hat{\xi}$. Consequently, we derive a range of "good" values for skeleton points. The first step is to use Laplace approximation to approximate the marginal likelihood $L_{\xi}\left(\sigma^{2} \mid \mathbf{y}\right)$ for given $\sigma^{2}$ and the second step is to integrate out $\sigma^{2}$ numerically, so the first step is nested within the second step. This method is presented below with further details in Appendix A.2.

First consider the integral in (8). Under the Gaussian prior assumption for $\beta$ we can derive the likelihood for $\sigma^{2}$ for given $\xi$ as,

$$
L_{\xi}\left(\sigma^{2} \mid \mathbf{y}\right)=\int_{\mathcal{R}^{n}} p\left[\mathbf{y}, \mathbf{z} \mid \sigma^{2}, \xi\right] \mathrm{d} \mathbf{z}
$$


where $p\left[\mathbf{y}, \mathbf{z} \mid \sigma^{2}, \xi\right]=p\left[\mathbf{y} \mid \boldsymbol{\mu}=f_{\nu}(\mathbf{z})\right] p\left[\mathbf{z} \mid \sigma^{2}, \xi\right]$, with $p\left[\mathbf{z} \mid \sigma^{2}, \xi\right]=\int_{\mathcal{R}^{p}} p\left[\mathbf{z} \mid \beta, \sigma^{2}, \xi\right] \pi(\beta) \mathrm{d} \beta$ being a Gaussian density.

Let

$$
\begin{aligned}
\tilde{\mathbf{z}}_{\xi}\left(\sigma^{2}\right) & =\underset{\mathbf{z}}{\operatorname{argmax}} p\left[\mathbf{y}, \mathbf{z} \mid \sigma^{2}, \xi\right], \\
\tilde{H}_{\xi}\left(\sigma^{2}\right) & =-\left.\frac{\partial^{2}}{\partial \mathbf{z} \partial \mathbf{z}^{\top}} \log p\left[\mathbf{y}, \mathbf{z} \mid \sigma^{2}, \xi\right]\right|_{\mathbf{z}=\tilde{\mathbf{z}}_{\xi}\left(\sigma^{2}\right)} .
\end{aligned}
$$

Then, by Laplace approximation (Barndorff-Nielsen and Cox, 1989),

$$
L_{\xi}\left(\sigma^{2} \mid \mathbf{y}\right) \approx p\left[\mathbf{y} \mid \boldsymbol{\mu}=f_{\nu}\left(\tilde{\mathbf{z}}_{\xi}\left(\sigma^{2}\right)\right)\right] p\left[\mathbf{z}=\tilde{\mathbf{z}}_{\xi}\left(\sigma^{2}\right) \mid \sigma^{2}, \xi\right]\left|\frac{1}{2 \pi} \tilde{H}_{\xi}\left(\sigma^{2}\right)\right|^{-\frac{1}{2}}
$$

for any given $\sigma^{2}$. Using this result in (8) we have

$m_{\xi}(\mathbf{y})=\int_{0}^{\infty} L_{\xi}\left(\sigma^{2} \mid \mathbf{y}\right) \pi\left(\sigma^{2}\right) \mathrm{d} \sigma^{2} \approx \int_{0}^{\infty} p\left[\mathbf{y} \mid \boldsymbol{\mu}=f_{\nu}\left(\tilde{\mathbf{z}}_{\xi}\left(\sigma^{2}\right)\right)\right] p\left[\mathbf{z}=\tilde{\mathbf{z}}_{\xi}\left(\sigma^{2}\right) \mid \sigma^{2}, \xi\right]\left|\frac{1}{2 \pi} \tilde{H}_{\xi}\left(\sigma^{2}\right)\right|^{-\frac{1}{2}} \pi\left(\sigma^{2}\right) \mathrm{d} \sigma^{2}$.

The integration in the right-hand side of (23) is done numerically using the trapezoid rule in a range of values of $\sigma^{2}$ where the integrand has significant mass.

Let $\tilde{m}_{\xi}(\mathbf{y})$ denote the approximation in (23). To derive a sensible region for the parameter $\xi$, let $\tilde{\xi}$ denote the maximizer of $\tilde{m}_{\xi}(\mathbf{y})$ and let $\tilde{m}_{\tilde{\xi}}(\mathbf{y})$ denote its maximum value. Suppose $\xi$ consists of

$d$ components. For each component $j$, we obtain an interval $\left(\xi_{j}^{L}, \xi_{j}^{U}\right)$ such that when $\xi_{j} \in\left(\xi_{j}^{L}, \xi_{j}^{U}\right)$ and the remaining components are equal to the corresponding components in $\tilde{\xi}, \tilde{m}_{\xi}(\mathbf{y})$ is no less than $\alpha \tilde{m}_{\tilde{\xi}}(\mathbf{y})$ for a predetermined factor $\alpha \in(0,1)$. Each interval is then discretized to a set of $T$ equispaced points $\left\{\xi_{j}^{1}=\xi_{j}^{L}, \xi_{j}^{2}, \ldots, \xi_{j}^{T}=\xi_{j}^{U}\right\}$ and the discrete points are crossed to create a finite grid of points $\left\{\xi_{1}^{1}, \xi_{1}^{2}, \ldots, \xi_{1}^{T}\right\} \times \ldots \times\left\{\xi_{d}^{1}, \xi_{d}^{2}, \ldots, \xi_{d}^{T}\right\}$. The points $\xi$ in this grid where $\tilde{m}_{\xi}(\mathbf{y})<\alpha \tilde{m}_{\tilde{\xi}}(\mathbf{y})$ are discarded and the remaining points, $\Xi$, define the skeleton set. If the number of points in $\Xi$ is deemed large for the available computational resources, then $\alpha$ is increased accordingly.

\subsection{Model choice and weighting}

In practice, information about the true underlying model is limited. Using parametric links can make inference more robust but this still assumes a specific parametric form for the link function and correlation function. So far we have discussed how to choose between models with the same parametric link and correlation function. In this section we discuss choosing between different links, and correlation families.

Suppose there are $R$ candidate models each of which specify a link and a correlation function, denoted by $\mathcal{M}_{r}\left(\xi_{r}\right)$, with associated parameters $\xi_{r}$, for $r=1, \ldots, R$. We write $p\left[\mathbf{y}, \mathbf{w} \mid \psi ; \mathcal{M}_{r}\left(\xi_{r}\right)\right]$ for the joint density of $\mathbf{y}$ and $\mathbf{w}$ under model $\mathcal{M}_{r}\left(\xi_{r}\right)$. Then, the corresponding marginal density for the data is

$$
m_{r, \xi_{r}}(\mathbf{y})=\int_{\mathcal{R}^{p} \times \mathcal{R}_{+}} \int_{\mathcal{R}^{n}} p\left[\mathbf{y}, \mathbf{w} \mid \psi ; \mathcal{M}_{r}\left(\xi_{r}\right)\right] \pi(\psi) d \mathbf{w} \mathrm{d} \psi .
$$

Although the marginal density of $\mathbf{y}$ remains the same whether integrated with respect to $\mathbf{w}$ or $\mathbf{z}$, we use the joint density of $\mathbf{y}$ and $\mathbf{w}$ because, later in this section, the RL estimation is used with reparameterized samples. Note that $\mathbf{w}$ is any transformed version of $\mathbf{z}$, so it can be $\mathbf{z}$ if we let $g_{\nu}$ to be the identity function. 
A general measure of model comparison is the AIC which is defined as

$$
\mathrm{AIC}_{r}=-2 \log m_{r, \hat{\xi}_{r}}(\mathbf{y})+2 d_{r}
$$

where $\hat{\xi}_{r}$ is the EB estimate of $\xi_{r}$ and $d_{r}$ is the number of parameters in $\xi_{r}$. A model with lower AIC value would be preferred, although it can also be used for model weighting in the spirit of Buckland et al. (1997), an approach we come to at the end of this section. The AIC formula is not straightforward to apply because we don't know the value of $m_{r, \hat{\xi}_{r}}(\mathbf{y})$. In Section 3.1 we have discussed how the RL method is used to estimate ratios $m_{r, \xi_{r}}(\mathbf{y}) / m_{r, \xi_{r, 1}}(\mathbf{y})$ for models having the same functional forms for the link function and the spatial covariance functions, i.e. within $\mathcal{M}_{r}$. Although, in principle, the GIS methods developed in Section 3.1 may be used to estimate Bayes factors across different models $\mathcal{M}_{r}$ 's, it is computationally demanding as large number of skeleton points with several combinations of $\xi_{r}$ values from these models need to be used for accurate estimation of Bayes factors. Instead, we consider minimizing

$$
\mathrm{AIC}_{r}^{*}=-2 \log \frac{m_{r, \hat{\xi}_{r}}(\mathbf{y})}{m_{1, \hat{\xi}_{1}}(\mathbf{y})}+2 d_{r},
$$

and apply the RL method to estimate the ratios $m_{r, \hat{\xi}_{r}}(\mathbf{y}) / m_{1, \hat{\xi}_{1}}(\mathbf{y})$ for $r=1, \ldots, R$.

Suppose $\left\{\psi^{(l, r)}, \mathbf{w}^{(l, r)}\right\}_{l=1}^{L_{r}}$ is a Harris ergodic Markov chain with stationary density $\pi\left(\psi, \mathbf{w} \mid \mathbf{y} ; \mathcal{M}_{r}\left(\hat{\xi}_{r}\right)\right)$ corresponding to the model $\mathcal{M}_{r}\left(\hat{\xi}_{r}\right), r=1, \ldots, R$. Let

$$
C_{r}=m_{r, \hat{\xi}_{r}}(\mathbf{y}) / m_{1, \hat{\xi}_{1}}(\mathbf{y}),
$$

and

where $L=\sum_{r} L_{r}$ and

$$
\eta_{r}=-\log C_{r}+\log \frac{L_{r}}{L}
$$

$$
P_{r}(\psi, \mathbf{w} ; \eta)=\frac{p\left[\mathbf{y}, \mathbf{w} \mid \psi ; \mathcal{M}_{r}\left(\hat{\xi}_{r}\right)\right] e^{\eta_{r}}}{\sum_{s=1}^{R} p\left[\mathbf{y}, \mathbf{w} \mid \psi ; \mathcal{M}_{s}\left(\hat{\xi}_{s}\right)\right] e^{\eta_{s}}},
$$

where $\eta=\left(\eta_{1}, \ldots, \eta_{R}\right)$. Estimation of $\eta$ is possible up to an additive constant using the samples $\left\{\psi^{(l, r)}, \mathbf{w}^{(l, r)}\right\}_{l=1}^{L_{r}}, r=1, \ldots, R$ by maximizing the quasi log-likelihood

$$
\mathcal{L}(\eta)=\sum_{r=1}^{R} \sum_{l=1}^{L_{r}} \log P_{r}\left(\psi^{(l, r)}, \mathbf{w}^{(l, r)} ; \eta\right) .
$$

Let $\hat{\eta}$ denote the maximizer of (24) subject to the constraint that $\sum \hat{\eta}_{r}=0$, and let $\hat{C}_{r}=\frac{L_{r}}{L} e^{-\hat{\eta}_{r}}$ be the corresponding estimate of $C_{r}$. Then, an estimate of $\mathrm{AIC}_{r}^{*}$ is

$$
\widehat{\mathrm{AIC}}_{r}^{*}=-2 \log \hat{C}_{r}+2 d_{r},
$$

and we choose the model with the smallest $\widehat{\mathrm{AIC}}_{r}^{*}$ value.

Instead of choosing a single model, Buckland et al. (1997) argue for an ensemble modeling approach where a model weight is calculated from the AIC values. In our case, we define the weight for the $r$ th model to be

$$
\text { Weight }_{r}=\frac{\exp \left(-\widehat{\mathrm{AIC}}_{r}^{*} / 2\right)}{\sum_{t=1}^{R} \exp \left(-\widehat{\mathrm{AIC}}_{t}^{*} / 2\right)}
$$

Let $\hat{\mu}_{r}(s)$ denote the estimated mean response at spatial location $s \in \mathbb{S}$ using the model $\mathcal{M}_{r}\left(\hat{\xi}_{r}\right)$, $r \in\{1, \ldots, R\}$. Then, an ensemble estimate of the mean response at that location is given by

$$
\hat{\mu}(s)=\sum_{r=1}^{R} \text { Weight }_{r} \times \hat{\mu}_{r}(s) .
$$




\section{Simulations}

In this section using simulation examples, we demonstrate the advantages of using transformed samples in GIS estimation. Simulation studies are also used to exhibit the performance of the proposed model selection criterion in choosing the true link functions and the spatial covariance structures. All analyses in this paper are performed using the R package geoBayes (Evangelou and Roy, 2018).

\subsection{Comparison with the untransformed estimator for the binomial-robit model}

The purpose of this section is to demonstrate that the GIS estimator based on the untransformed samples (method of Roy et al. (2016)) can be biased for estimating $\xi$ when the skeleton set is not dense enough. The reason for this bias is the little "overlap" among the importance densities. On the other hand, the reparameterized version (15), although unbiased, is much slower because computing the robit link function for degrees of freedom $\nu<1$ is slow. Instead, (17) with the Wallace link reparameterization (3) provides unbiased estimates, and is also faster than (15).

We consider the spatial domain $\mathbb{S}=[0,1] \times[0,1]$ and randomly select $n=100$ locations $s \in \mathbb{S}$ to sample from. The spatial random field $z(s)$ is assumed to have exponential correlation structure with unknown spatial range parameter $\phi=0.5$ and variance $\sigma^{2}=1$. The mean of the random field is taken to be constant $\beta=-1$.

In this section the response variable is conditionally binomially distributed given the value of the spatial field with number of trials $t=100$ at each sampling location and the probability of success at location $s, \mu(s)$, is given by

$$
\mu(s)=G_{\nu}(z(s))
$$

where $G_{\nu}(\cdot)$, as defined in section 2 , is the cdf of the standard Student's $t$ distribution with $\nu$ degrees of freedom. Here we take $\nu=0.5$ for simulating the data. The parameters $\beta$ and $\sigma^{2}$ are assigned normal and scaled-inverse-chi-square priors as discussed in Section 3 with hyperparameter values $m_{b}=0, V_{b}=100, n_{\sigma}=1$, and $a_{\sigma}=1$. The link parameter $\nu$ and spatial range parameter $\phi$ are then treated as unknown and are estimated using the EB procedure of section 3 . The skeleton set for the parameters $\xi=(\nu, \phi)$ is set to

$$
\Xi=\{0.4,1,3,7,14\} \times\{0.25,0.7,1\} .
$$

For fixed $\xi \in \Xi$, we sample from $\pi_{\xi}\left(\beta, \sigma^{2}, \mathbf{z} \mid \mathbf{y}\right)$, the complete posterior density of $\beta, \sigma^{2}$ and the random field $\mathbf{z}$ conditional on the observed data $\mathbf{y}$. For each $\xi \in \Xi$, we obtain a Markov chain sample of size 1000 after a burn-in of 300 samples and thinning of 5. From these samples, 800 samples were used to obtain RL estimate $\hat{\mathbf{r}}$, and the remaining 200 samples were used to calculate the GIS estimator $B_{\xi, \xi_{1}}(\hat{\mathbf{r}})$. We computed the three GIS estimators given in (10), (15), and (17). Once $\hat{\xi}$ is estimated using these GIS estimators, posterior means of the parameters $\left(\beta, \sigma^{2}\right)$ are estimated based on Markov chain samples of length 1000 after a burn-in of 300 samples and thinning of 5 from the posterior density $\pi_{\hat{\xi}}(\psi \mid \mathbf{y})$.

We performed 100 simulations where the sampling locations remained the same but the spatial random field and the observations were different. In Figure 1 we show the kernel density of the parameter estimates using each of the three methods described in this paper. It is clear that the GIS estimator (10) with untransformed samples can lead to incorrect inference while the two methods based on the transformed samples do not exhibit such bias. The exact biases are shown in Table 1 along with the mean square difference from the true value of the spatial field, which also shows that the untransformed estimator has the worst performance. On the other hand, as shown in Table 1, using the link transformation (15) can be slow for $\nu<1$. The alternative transformation method (17) is much faster although perform similarly as (15). 

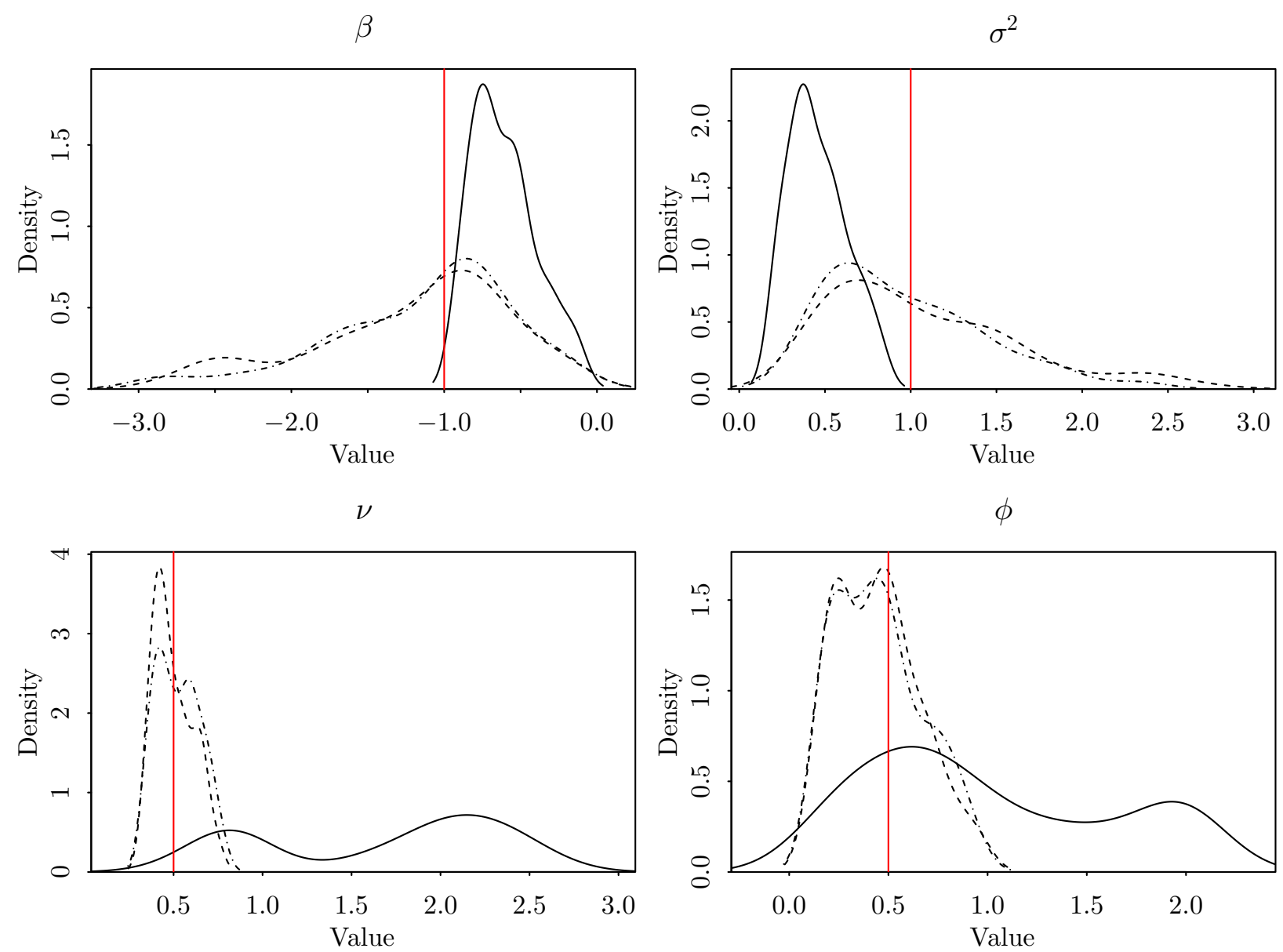

Figure 1: Distribution of parameter estimates over all simulations for the binomial robit model using different transformations based GIS: None (solid); Link (dashed); Wallace (dashed-dotted). The true parameter value is shown by a vertical line.

\begin{tabular}{lccccccc}
\hline & $\operatorname{Bias}(\nu)$ & $\operatorname{Bias}(\phi)$ & $\operatorname{Bias}(\beta)$ & $\operatorname{Bias}\left(\sigma^{2}\right)$ & $\operatorname{MSE}(\mathbf{z})$ & Time 1st stage & Time 2nd stage \\
\hline None & 1.17 & 0.50 & 0.39 & -0.55 & 0.50 & 28 & 61 \\
Link & 0.00 & -0.05 & -0.22 & 0.06 & 0.29 & 96 & 315 \\
Wallace & 0.02 & -0.05 & -0.16 & -0.05 & 0.27 & 38 & 114 \\
\hline
\end{tabular}

Table 1: Bias of the empirical Bayes estimates for $\nu$ and $\phi$, bias of the posterior estimates of $\beta$ and $\sigma^{2}$, mean square error of the posterior for $\mathbf{z}$, and computing times in seconds for the first and second stage per iteration for the simulation example of Section 4.1 


\begin{tabular}{lccccccccc}
\hline Link & robit & probit & mGEV & robit & probit & mGEV & robit & probit & mGEV \\
Correlation & exp & exp & exp & Gau & Gau & Gau & spher & spher & spher \\
\hline M1 (robit, exp) & 0.36 & 0.02 & 0.08 & 0.03 & 0.06 & 0.03 & 0.32 & 0.02 & 0.08 \\
M2 (mGEV, spher) & 0.05 & 0.07 & 0.32 & 0.00 & 0.00 & 0.00 & 0.07 & 0.08 & 0.41 \\
\hline
\end{tabular}

Table 2: Average model weight for each model given in the columns using data generated from the model given in the rows.

\subsection{Model selection}

In this section we demonstrate that the model selection criterion of Section 3.3 chooses the correct model for the link and correlation functions. We randomly choose $n=100$ locations to sample from within the spatial domain $\mathbb{S}=[0,1] \times[0,1]$. Samples are taken from two models. Model M1 is the binomial SGLMM with robit link and exponential correlation, and model M2 is the binomial SGLMM with modified GEV link and spherical correlation. For both models the spatial random field was sampled with spatial range $\phi=0.5$, relative nugget $\omega=0$ (assumed known), variance $\sigma^{2}=1$, and mean $\beta=0.5$. Conditioned on the value of the spatial field, the observation at the $i$ th location was sampled from the binomial distribution with number of trials $t_{i}=100$ for all $i$ and probability of success $F_{\nu}\left(z_{i}\right)$ where $F_{\nu}(\cdot)$ is the cdf of the standard Student's $t$ distribution with degrees of freedom $\nu=0.5$ for model M1, and the function in (5) with $\nu=0$ for model M2. Our models are completed by assuming a scaled-inverse-chi-squared prior for $\sigma^{2}$ with degrees of freedom 1 and scale 1 , and a conditional normal prior for $\beta$ given $\sigma^{2}$ with mean 0 and variance $10 \sigma^{2}$.

From each model, 100 different data sets were simulated from the same 100 locations but with different spatial random field each time. For each simulated data set, we fit nine different models by assuming three different link functions: robit, probit, and modified GEV, combined with three different correlation functions: exponential, Gaussian, spherical. The skeleton set for $\xi=(\nu, \phi), \Xi$, for each model corresponds to $\Xi=\Xi_{\nu} \times \Xi_{\phi}$ where $\Xi_{\nu}=\{0.4,1,3,7,15\}$ for the robit link, $\Xi_{\nu}$ is the null set for the probit link, $\Xi_{\nu}=\{0,0.5,1\}$ for the modified GEV link, and $\Xi_{\phi}=\{0.3,0.7,1.1\}$

for all three correlation functions considered. We fit each model by first estimating $\xi$ by $\hat{\xi}$ from maximizing the reparameterized estimator (15), and then calculating each model's weight using (25). The estimation of $\xi$ is done by generating MCMC samples from the posterior distribution of $(\psi, \mathbf{z})$ conditioned on a value of $\xi$ in the skeleton set. The size of MCMC samples, after a burn in of 300 samples, is $10^{4} / k$ rounded down where $k$ is the size of the skeleton set. From these samples approximately $80 \%$ is used for stage 1 , and the remaining samples are used for stage 2 of the procedure described in Appendix B. To estimate the model weights we use 1000 MCMC samples, after a burn in of 300 samples, from the posterior density of $(\psi, \mathbf{z})$ conditioned on the value of $\hat{\xi}$ from each model.

The average weight for each model over the 100 different simulated datasets is given in Table 2 . In both cases the correct model has the highest average weight. The results show that the proposed approach is very good in selecting the correct link function among those considered. Due to the similarities between the exponential and spherical correlations (Stein, 1999, Sec 2.10) the secondbest model chooses either of these when the true model is the other, however, the Gaussian model, which is not close to the true model, is not favored by our criterion. 


\section{Examples with real data analysis}

This section illustrates the proposed link functions, reparameterized GIS estimators and the EB methodology using binomial and Poisson SGLMMs fitted to analyze two real spatial data sets.

\subsection{Analysis of radionuclide concentrations on the Rongelap island}

The dataset consists of the measurements of $\gamma$-ray counts $y_{i}$ observed during $t_{i}$ seconds at $i$ th coordinate on the Rongelap island, $i=1, \ldots, n, n=157$. This data set was analyzed by Diggle et al. (1998) and Christensen (2004), among others, using a Poisson spatial model. Using likelihood analysis, Christensen (2004) found that the Box-Cox link with $\nu=0.84$ was more appropriate for these data if an exponential correlation is used. Here we demonstrate the application of EB methodology developed in section 3 on this example.

Our model consists of a Poisson SGLMM with the modified Box-Cox link function for the $\gamma$-ray counts. For the spatial Gaussian random field we fit a constant mean $\beta$ and covariance consisting of a partial sill parameter $\sigma^{2}$, a relative nugget term $\omega$, and a correlation function parameterized by $\theta$, which is yet to be determined. The parameters $\beta$ and $\sigma^{2}$ are assigned the conditional normal and scaled-inverse-chi-square priors of Section 3 respectively with $m_{b}=0, V_{b}=100, a_{\sigma}=1$, and $n_{\sigma}=1$. In addition, the parameters $\xi=(\nu, \omega, \theta)$ are also unknown and are estimated by the EB estimate $\hat{\xi}$ from maximizing (15).

We consider four different models for the correlation function: Matérn, exponential-power, spherical, and exponential. All families contain a spatial range parameter $\phi$ while the first two contain an additional parameter $\kappa$.

At the first stage, we seek a set of skeleton points for the computation of the GIS estimators. For this we maximize the approximate marginal likelihood as discussed in Section 3.2. The maximizer, $\tilde{\xi}$, for each model can be seen in Figure 2, Next we explore the likelihood for a range of values of $\xi$ around $\tilde{\xi}$. Initially we fix all but one of the components of $\xi$ at $\tilde{\xi}$ and vary the other one widely. This allows us to compute the approximate likelihood quickly for a wide range of each parameter. Then we focus on a narrower range of the parameters where the marginal likelihood value is at least $60 \%$ from its maximum (see Figure 2 2 ). We choose $T=3$ with the notation of Section 3.2 and evaluate the approximate likelihood at each combination of parameter values in this narrower range, again discarding any combinations whose approximate likelihood value falls below the $60 \%$ threshold. The remaining combinations were used as skeleton points for the GIS estimators. After this procedure we were left with $12,10,5$, and 4 skeleton points for the four models respectively, listed in Appendix C. Table 8. (Note that the third and fourth models have one less parameter.)

For each set of parameters $\xi$ in the skeleton set, we draw MCMC samples from the posterior density $\pi_{\xi}\left(\beta, \sigma^{2}, \mathbf{z} \mid \mathbf{y}\right)$ of the parameters $\beta, \sigma^{2}$ and the spatial field $\mathbf{z}$. The MCMC was run with burn-in 300, while retaining a sample of size $N / k$ (rounded down) where $N=50000$ and $k$ is the number of skeleton points for each model given in the previous paragraph. Thus the total number of samples used in the procedure of Appendix B for each model is about the same. We use approximately $80 \%$ of the samples for Stage 1 and the remaining $20 \%$ of the samples for Stage 2 . The estimates $\hat{\xi}$ for each model are shown in Table 3 . We also provide standard errors for the EB estimate $\hat{\xi}$ obtained by the method described in Appendix A.1.

Subsequently, we fix the parameters $\xi$ at $\hat{\xi}$, and take a new MCMC sample with burn-in 300, and size 5000. The new sample is used to estimate the mean parameter $\beta$, the partial sill parameter $\sigma^{2}$, and predict the spatial field. Examination of the posterior samples showed no significant autocorrelations. The posterior means for the two parameters are also shown in Table 3. The batch means estimates of standard errors for the posterior mean estimates are also provided. 

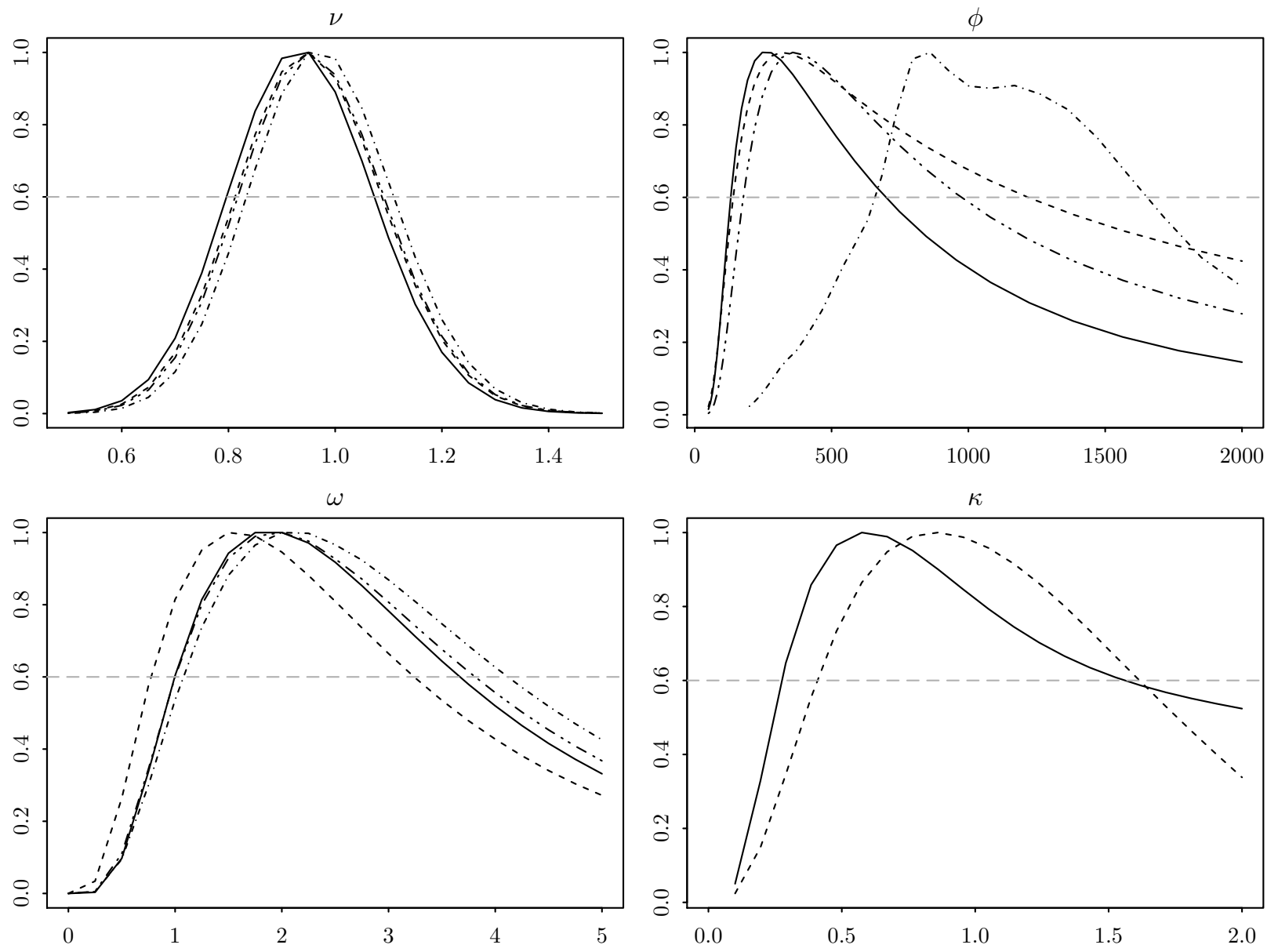

Figure 2: Approximate likelihood computed for a range of parameter values for the Rongelap example. The parameter on the horizontal axis varies while the other parameters remain fixed at their estimates $\tilde{\psi}$. A narrower range is then considered such that the value of the likelihood is at least $60 \%$ from its maximum. The four models for the correlation function considered are: Matérn (solid); exponential-power (dashed); spherical (dashed-dotted); exponential (dashed-dotted-dotted). 


\begin{tabular}{lllllllllc}
\hline Model & $\beta$ & $\sigma^{2}$ & $\nu$ & $\phi$ & $\omega$ & $\kappa$ & $\log \mathrm{BF}$ & $|\xi|$ & Weight \\
\hline Matérn & 5.288 & 2.083 & 0.963 & 324 & 2.211 & 0.637 & 0 & 4 & 0.136 \\
& $(0.502)$ & $(0.239)$ & $(0.146)$ & $(420)$ & $(1.847)$ & $(0.985)$ & & & \\
Exp-power & 5.856 & 2.134 & 0.966 & 393 & 2.178 & 1.096 & -0.007 & 4 & 0.135 \\
& $(0.500)$ & $(0.247)$ & $(0.146)$ & $(336)$ & $(1.957)$ & $(0.917)$ & & & \\
Spherical & 5.955 & 1.959 & 0.978 & 1170 & 2.598 & & -0.020 & 3 & 0.363 \\
& $(0.525)$ & $(0.220)$ & $(0.141)$ & $(332)$ & $(1.810)$ & & & & \\
Exponential & 5.780 & 2.129 & 0.957 & 384 & 2.065 & & -0.014 & 3 & 0.365 \\
& $(0.501)$ & $(0.244)$ & $(0.145)$ & $(324)$ & $(1.501)$ & & & & \\
\hline
\end{tabular}

Table 3: Parameter estimates with standard errors and log Bayes factor relative to the Matérn model for the Rongelap example for each model. The standard errors estimates are provided in parentheses. The size of $\xi$ is denoted by $|\xi|$.

Using the new MCMC samples, we also compute the Bayes factors for the three models relative to the Matérn model as discussed in Section 3.3. The estimates of the Bayes factors are shown in Table 3. It can be seen that the four models have about equal Bayes factors. The exponential and spherical models have one fewer parameter, so they are preferable. The estimate of $\nu$ is slightly higher than Christensen's (2004) estimate $(\nu=0.84)$, and significantly different from the log link $(\nu=0)$ used in Diggle et al. (1998). We also provide the weight of each model as given by (25), and use that to calculate ensemble average estimates of the predicted rate according to (26).

Using the new MCMC samples, we consider prediction of the Poisson rate (per unit time) at 1709 locations covering the island using the four candidate models and also the ensemble prediction given in (26). These predictions are shown in Figure 3, along with the observed count per unit time. It can be seen that the predicted Poisson rate has similar pattern for all models, with higher values at the west side of the island, and matches that of the observed data closely. Examination of the range of prediction across all locations shows that the exponential model has the highest range (4.9 to 10 ), followed by the exponential-power (5 to 9.9), the Matérn (5.1 to 9.9), and the spherical model (5.4 to 9.8), while the ensemble model's range is 5.2 to 9.9 . The prediction standard deviation falls in the range of 2.3 to 2.7 for all models.

To assess the predictive performance of each model, we performed leave-one-out crossvalidation. For each $i=1, \ldots, n$, the observation $y_{i}$ was deleted from the data set, and each model was fitted to the remaining data using the same two-stage procedure described in the beginning of this section. Let $\mu_{i}$ denote the mean per unit time at the location of the deleted observation, $\mathbf{y} \backslash i_{i}$ the vector of observations without $y_{i}$, and $\hat{\xi}_{\backslash i}$ the estimate of $\xi$ from the first stage using data $\mathbf{y}_{\backslash i}$. At the end of the first stage, a MCMC sample $\mu_{i}^{(1)}, \ldots, \mu_{i}^{(L)}$, with $L=1000$ after a burn in of 300 samples, was obtained from the conditional distribution of $\mu_{i}$ given $\left(\mathbf{y}_{\backslash i}, \hat{\xi}_{\backslash i}\right)$. We evaluate each model, as well as the ensemble model, by calculating its average negative predictive score given by

$$
\text { NegScore }=-\frac{1}{L} \sum_{l=1}^{L} \sum_{i=1}^{n} \log p\left[y_{i} \mid \mu_{i}^{(l)}\right],
$$

where $p\left[y_{i} \mid \mu_{i}\right]$ denotes the Poisson pmf with rate $t_{i} \mu_{i}$ evaluated at $y_{i}$. The model with the lowest negative score is preferred. We also calculate the average root mean square error given by

$$
\mathrm{RMSE}=\sqrt{\frac{1}{L} \sum_{l=1}^{L} \sum_{i=1}^{n}\left(y_{i}-t_{i} \mu_{i}^{(l)}\right)^{2}} .
$$




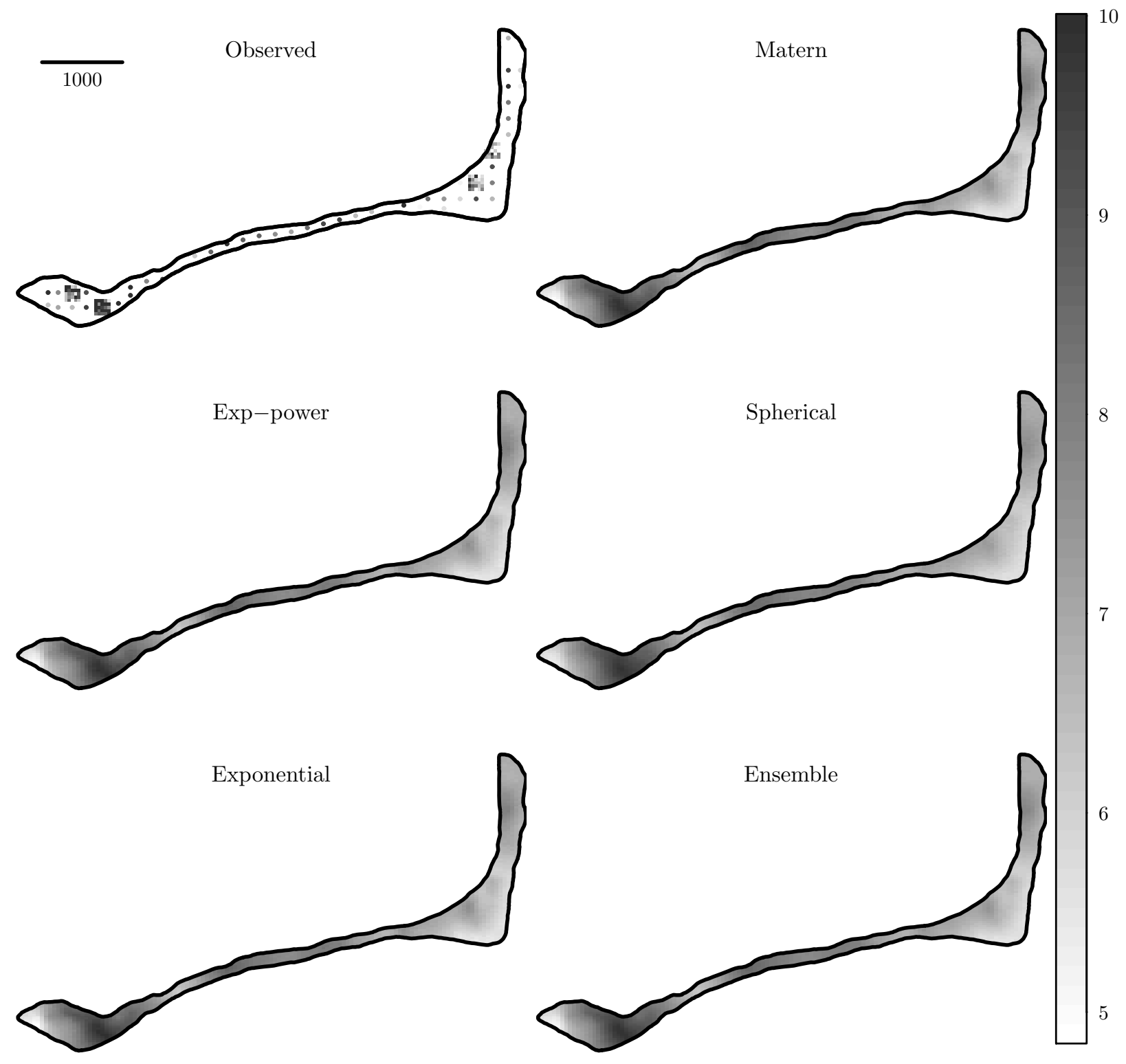

Figure 3: Observed count per unit time and prediction of the Poisson rate (per unit time) for the Rongelap example under four different models and ensemble prediction. 


\begin{tabular}{lrrrrr}
\hline & Matérn & Exp-power & Spherical & Exponential & Ensemble \\
\hline NegScore & 63976 & 64923 & 63896 & 62482 & 36797 \\
RMSE & 21359 & 21499 & 21406 & 21185 & 17329 \\
\hline
\end{tabular}

Table 4: Negative score and RMSE for the models used in the analysis of the Rongelap example. Smaller values are preferred.

The model with the lowest RMSE is preferred. The results are shown in Table 4 . The results show that the exponential model has the best predictive performance, and the exponential-power model has the worst performance. The weights given in Table 3 agree with this ranking. The ensemble model is significantly better than any of the individual models.

\subsubsection{Comparison with a fully-Bayesian approach}

An alternative to our EB method is a fully-Bayesian (FB) analysis. In FB method, the components of $\xi$ are also assigned priors together with the priors on $\psi$. Also, in this case, MCMC algorithms are used to obtain samples from the joint posterior distribution of $\mathbf{z}, \psi$ and $\xi$. Sampling from this posterior distribution can be difficult because of the correlation between the parameters (Christensen et al., 2006). In this section we apply a FB approach to the Rongelap data and compare it with the method proposed in this paper. Since we have identified that the model with the exponential correlation provides the best fit for these data, we focus on this model. We also fix the link function parameter to its estimate $\nu=0.957$, as the choice of an appropriate prior on this degrees of freedom parameter is known to be problematic (Doss (2012, p. 20), Roy (2014, p. 99-100)).

Previously, we used Laplace approximation to identify a suitable range for $\phi$ within $(178,975)$ and for $\omega$ within $(1.00,3.82)$ (see Figure 2). We consider two different FB models depending on the choice of prior. Model FB1 assumes independent uniform priors using the information of these ranges, i.e., $\pi(\phi) \propto 1_{(178,975)}(\phi)$ and $\pi(\omega) \propto 1_{(1.00,3.82)}(\omega)$. Mimicking the scale invariant prior $\pi(\phi) \propto 1 / \phi$, model FB2 assumes $\pi(\phi) \propto \phi^{-1} 1_{(0,2000)}(\phi)$ and $\pi(\omega) \propto \omega^{-1} 1_{(0,5)}(\omega)$. The other parts of the model remained the same.

Using trial MCMC runs, we selected Metropolis-Hastings steps (with joint updates for $(\phi, \omega)$ ) so that the acceptance rate is about 25\%. A total of 55000 MCMC samples, after a burn in of 300 samples, were selected from the posterior distribution of $(\mathbf{z}, \psi, \xi)$ given the data. The total MCMC sample size matches the one from the EB analysis.

In terms of computing time, the FB methods were slower: FB1 took 89 seconds, FB2 took 106 seconds, and the proposed EB took 48 seconds but with the additional overhead of having to estimate $\nu$. Plots of the posterior densities for the parameters using each method are shown in Figure 4. Although the posterior density for $\beta$ is similar using either method, the posterior for $\sigma^{2}$ has higher variance with the two FB approaches compared to the EB approach, which is not surprising given that FB analysis also samples $\phi$ and $\omega$. Furthermore, the posteriors for $\phi$ and $\omega$ in the case of FB1 are not very informative, and are different from the posteriors under FB2. This shows that the results are sensitive to the choice of prior, something which the EB approach avoids. The autocorrelation plots (Figure 5) show that the MCMC chains (except for the $\beta$ chain) for the FB models suffer from high lag covariances. One of the reasons for slow mixing of the Markov chains in the FB models is the strong (posterior) correlation between the parameters, for example, the correlation between $\sigma^{2}$ and $\omega$ is -0.9 for FB1 and -0.8 for FB2. The mixing of the MCMC algorithms for FB models may be improved by reparameterization (Christensen et al., 2006), although it is unclear how this can be implemented for the general models considered in this 
$\beta$

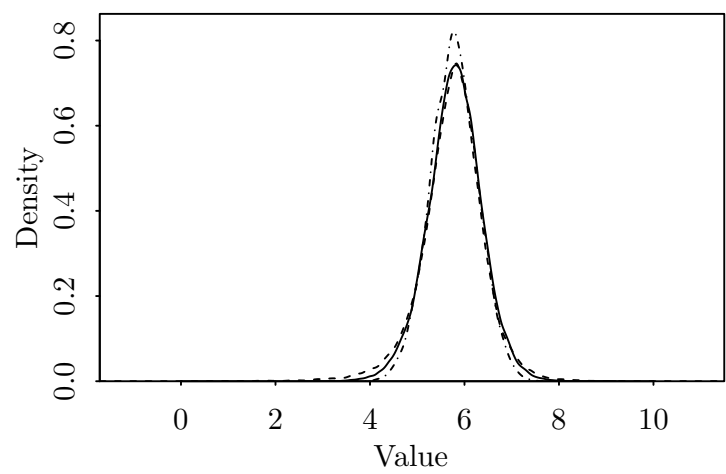

$\phi$

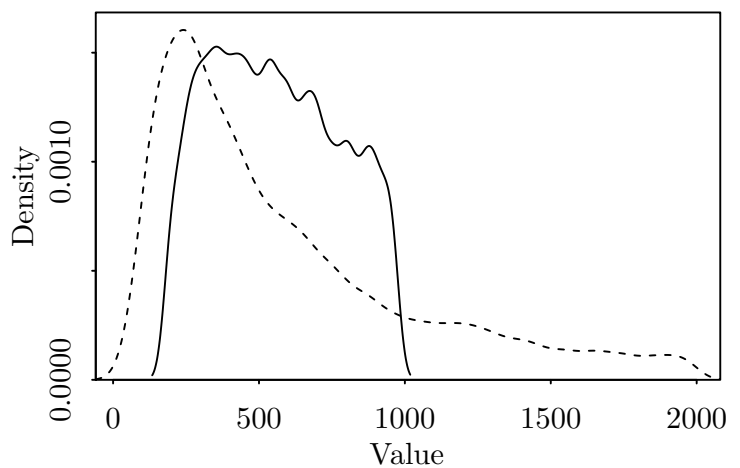

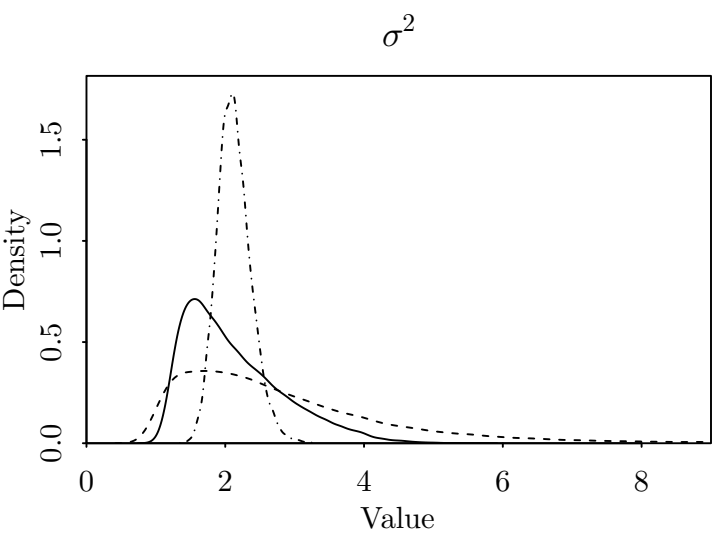

$\omega$

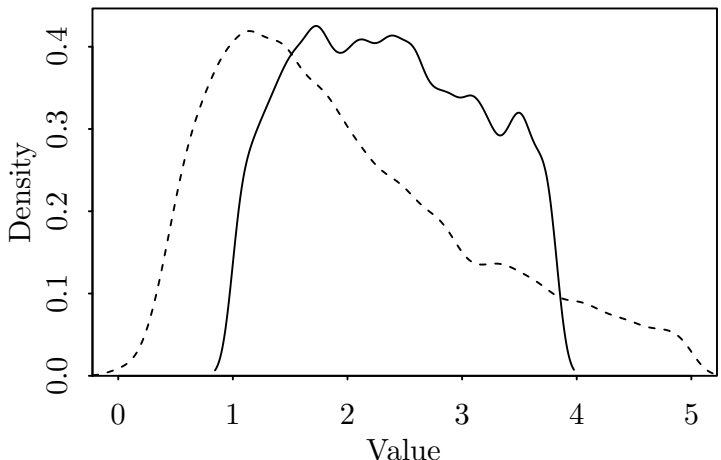

Figure 4: Posterior densities for the two fully-Bayesian approaches, FB1 (solid line) and FB2 (dashed line), and the proposed empirical Bayes approach (dashed-dotted line) for the Rongelap example.

paper.

\subsubsection{Comparison with the untransformed estimator and separability of the Box-Cox model}

As discussed in Section [3.1, if the skeleton set is chosen sparsely, then the Monte-Carlo sample can become separable. This phenomenon is particularly acute when the link function changes significantly for small changes of its parameter, which is the case for the Box-Cox link. In fact, for this model the MCMC sample can be completely separable, therefore the RL estimator (12) is unidentifiable when using the untransformed samples. Consequently, the estimator (10) is undefined and so is the EB estimate for $\xi$.

We focus on estimation of the link function parameter only by maximizing the estimated BF's because the separability issue arises when the link function parameter varies. We use the Poisson modified Box-Cox model with exponential correlation, and fix the covariance parameters at $\phi=400$ and $\omega=2.2$. The prior distributions for $\beta$ and $\sigma^{2}$ remain unchanged from our original analysis of these data. The skeleton set for $\nu$ is set to $\Xi=\left\{\xi_{1}, \xi_{2}, \xi_{3}\right\}=\{0.8,1.0,1.2\}$. Despite these values chosen to be very close, we will see that the untransformed estimator fails to estimate the BF between these three models accurately.

For each value of $\nu \in \Xi$, we take an MCMC sample from the posterior distribution of $(\psi, \mathbf{z})$ of length 1300 out of which the first 300 samples are discarded and the final $N=1000$ samples are 

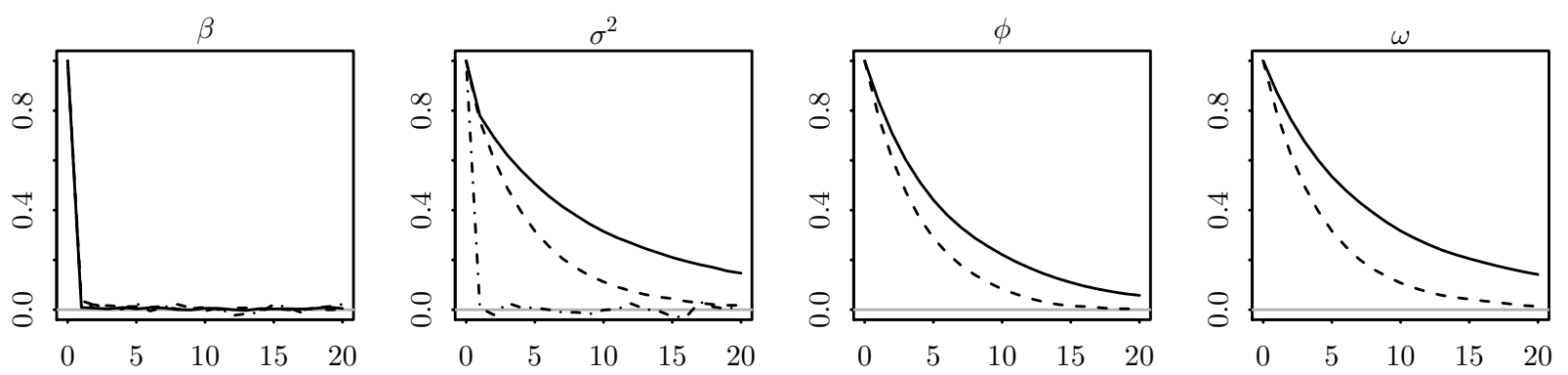

Figure 5: Autocorrelation plots of the MCMC samples for the two fully-Bayesian approaches, FB1 (solid line) and FB2 (dashed line), and the proposed empirical Bayes approach (dashed-dotted line) for the Rongelap example.

retained. Let $\left(\psi^{(j ; l)}, \mathbf{z}^{(j ; l)}\right)$ denote the $l$ th sample from $\pi_{\xi_{j}}(\psi, \mathbf{z} \mid \mathbf{y})$ when $\nu=\xi_{j}$, for $l=1, \ldots, N$, $j=1,2,3$. Also let $\mu^{(j ; l)}=h_{\nu_{j}}^{-1}\left(\mathbf{z}^{(j ; l)}\right), l=1, \ldots, N$ be the transformed posterior samples for the mean, that is, from $\pi_{\xi_{j}}(\psi, \boldsymbol{\mu} \mid \mathbf{y})$ given in (14). The RL estimator can be evaluated using either $\mathbf{z}^{(j ; l)}$ or $\mu^{(j ; l)}$ samples. The quasi log likelihood, which is maximized to obtain the RL estimator is defined in terms of the sample inclusion probabilities $\tilde{p}_{j}$ 's in (13) which in turn is defined in terms of the likelihood

$$
L_{\mathbf{z}}^{(i ; j ; l)}=p\left[\mathbf{y} \mid \boldsymbol{\mu}=f_{\nu_{i}}\left(\mathbf{z}^{(j ; l)}\right)\right] p\left[\mathbf{z}^{(j ; l)} \mid \psi^{(j ; l)}, \nu_{i}\right]
$$

when using $\mathbf{z}^{(j ; l)}$ samples and

$$
L_{\mu}^{(i ; j ; l)}=p\left[h_{\nu_{j}}\left(\mu^{(j ; l)}\right) \mid \psi^{(j ; l)}, \nu_{i}\right] \tilde{J}_{\nu_{i}}\left(\mu^{(j ; l)}\right),
$$

when using $\mu^{(j ; l)}$ samples. The separability issue discussed in Gever's (1994) arises if there exists a partition $\Xi_{1}, \ldots, \Xi_{m}$ of skeleton points $\Xi$ such as for each $(j ; l)$, there exists $r \in\{1, \ldots, m\}$ such that $\xi_{i} \notin \Xi_{r}$ implies $L^{(i ; j ; l)}=0$. In this case the Bayes factors can be estimated for densities within the same partition but not between partitions. For the chosen model, separability is mathematically impossible, but can happen numerically if the corresponding observed (Markov chain) sample and the Poisson rate parameter are very different.

Figure 6 shows plots of $\log$-likelihood values $\left(\log L_{\mathbf{z}}^{(i ; j ; l)}\right)$ plotted against $\log L_{\mathbf{z}}^{\left(i^{\prime} ; j ; l\right)}$ for $i \neq i^{\prime}$ in the upper triangle. The colors correspond to each $j$. Similarly in the lower triangle we plot $\log L_{\mu}^{(i ; j ; l)}$ against $\log L_{\mu}^{\left(i^{\prime} ; j ; l\right)}$. It can be seen (Table 5) that the $\log L_{\mathbf{z}}^{(i ; j ; l)}$ are very different for different $i$ and their differences are in the range of tens of thousands so when taking exponentials, it will yield a zero. (The inclusion probabilities $\tilde{p}_{j}$ 's in (13) depend on the ratio of likelihoods $L_{\mathbf{z}}^{(i ; j ; l)}$, s which is equivalent to exponential of differences of log-likelihoods.) On the other hand, the differences for the transformed sample are in the range of ones so the reparameterized sample does not suffer from the separability issue. Thus, when using the likelihood from the untransformed samples, $L_{\mathbf{z}}^{(i ; j ; l)}$, it is impossible to estimate $\nu$ because the $\mathrm{BF}$ estimators are unidentifiable.

\subsection{Analysis of the incidence rates of the Rhizoctonia root rot}

In this example we analyze the root infection rates caused by Rhizoctonia fungi on wheat and barley. Data were collected at 100 locations where 15 plants were pulled out at each location and the total number of crown roots and infected crown roots were counted. These data were originally analyzed by Zhang (2002) who used a binomial SGLMM with logit link and spherical correlation. In this 


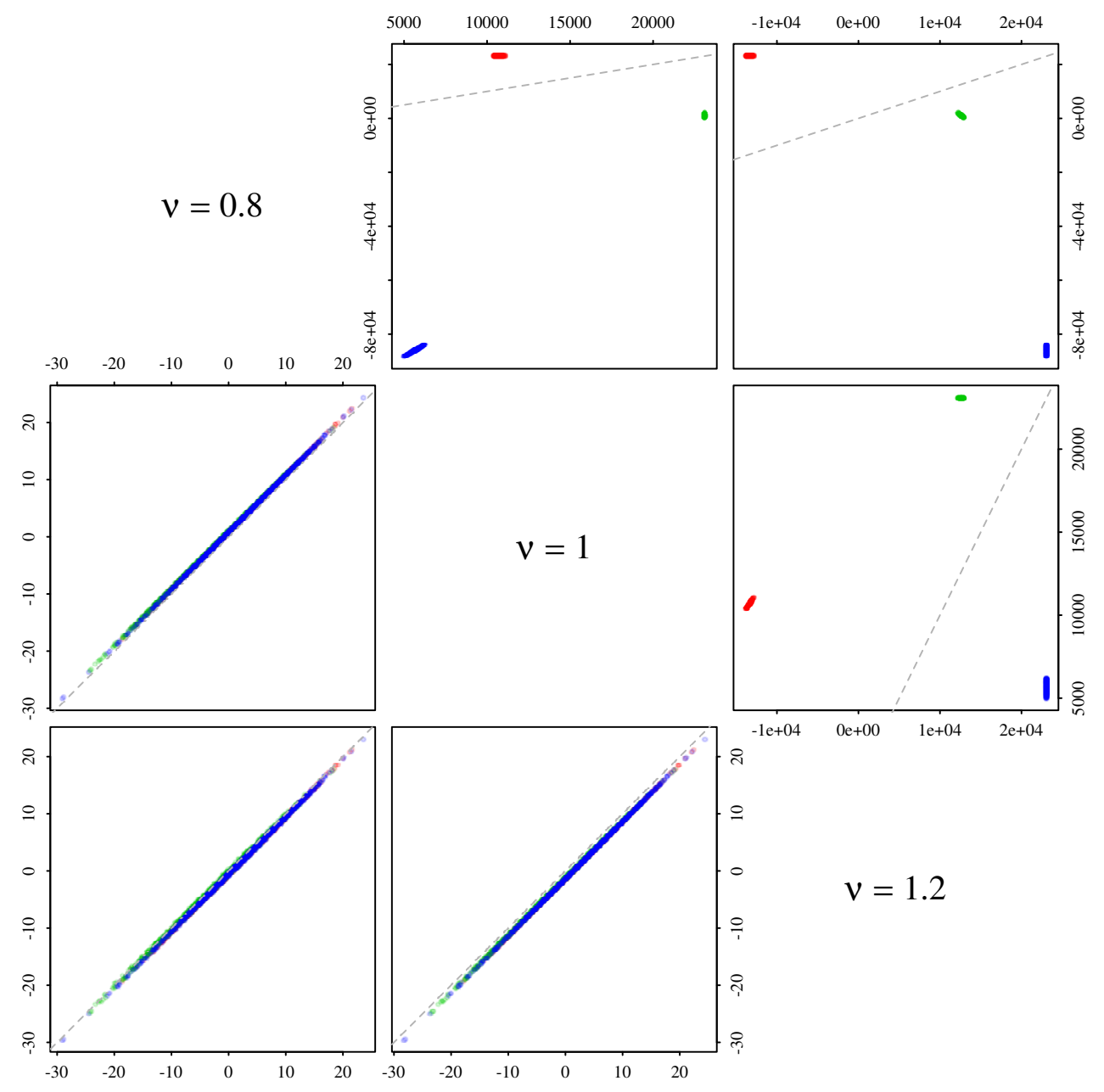

Figure 6: Log-likelihood values evaluated at different link parameters as indicated in the diagonal evaluated at data generated from the posterior distribution with link parameter: $\nu=\xi_{1}$ (red), $\nu=\xi_{2}$ (green), $\nu=\xi_{3}$ (blue) and plotted against each other. The upper triangle shows the loglikelihoods for the untransformed samples and the lower triangle shows the log-likelihoods for the transformed samples. The dashed line corresponds to the line with slope 1 and intercept 0 .

\begin{tabular}{cccc}
\hline & 1 & 2 & 3 \\
\hline $1-2$ & $(1.2 \mathrm{e}+4,1.3 \mathrm{e}+4)$ & $(-2.3 \mathrm{e}+4,-2.1 \mathrm{e}+4)$ & $(-9.3 \mathrm{e}+4,-9.0 \mathrm{e}+4)$ \\
$1-3$ & $(3.6 \mathrm{e}+4,3.7 \mathrm{e}+4)$ & $(-1.3 \mathrm{e}+4,-1.0 \mathrm{e}+4)$ & $(-1.1 \mathrm{e}+5,-1.1 \mathrm{e}+5)$ \\
$2-3$ & $(2.4 \mathrm{e}+4,2.4 \mathrm{e}+4)$ & $(1.0 \mathrm{e}+4,1.1 \mathrm{e}+4)$ & $(-1.8 \mathrm{e}+4,-1.7 \mathrm{e}+4)$ \\
\hline
\end{tabular}

\begin{tabular}{cccc}
\hline & 1 & 2 & 3 \\
\hline $1-2$ & $(-1.0,-0.6)$ & $(-1.3,-0.6)$ & $(-1.0,-0.6)$ \\
$1-3$ & $(0.1,1.0)$ & $(-0.4,0.9)$ & $(0.1,1.0)$ \\
$2-3$ & $(1.1,1.6)$ & $(0.9,1.5)$ & $(1.2,1.6)$ \\
\hline
\end{tabular}

Table 5: Top: Range of differences $\log L_{\mathbf{z}}^{(i ; j ; l)}-\log L_{\mathbf{z}}^{\left(i^{\prime} ; j ; l\right)}$ for $\left(i, i^{\prime}\right)$ shown in the rows for each $j$ shown in the columns. Bottom: The same for $\log L_{\mu}^{(i ; j ; l)}-\log L_{\mu}^{\left(i^{\prime} ; j ; l\right)}$. 


\begin{tabular}{llllllll}
\hline Model & $\beta$ & $\sigma^{2}$ & $\nu$ & $\phi$ & $\log$ BF & $|\xi|$ & Weight \\
\hline Robit, Spherical & -0.983 & 8.232 & $\infty$ & 3113 & 0 & 1 & 0.192 \\
& $(2.344)$ & $(1.448)$ & & $(2063)$ & & & \\
Robit, Exponential & -0.997 & 7.299 & $\infty$ & 1848 & 0.246 & 1 & 0.245 \\
& $(2.277)$ & $(1.201)$ & & $(1248)$ & & & \\
Modified-GEV, Spherical & -0.619 & 8.275 & 0.067 & 4367 & 0.660 & 2 & 0.136 \\
& $(2.525)$ & $(1.388)$ & $(0.327)$ & $(3332)$ & & & \\
Modified-GEV, Exponential & -0.620 & 7.710 & 0 & 2922 & 0.801 & 1 & 0.427 \\
& $(2.428)$ & $(1.312)$ & & $(1946)$ & & & \\
\hline
\end{tabular}

Table 6: Parameter estimates and log Bayes factor relative to the Matérn model for the Rhizoctonia example for each model. The size of $\xi$ is denoted by $|\xi|$ (counting only the components whose estimates fall in the interior of the parameter space).

paper we consider four different models. The link function is chosen among a robit or modified GEV link and the correlation function is chosen among a spherical or exponential model. Thus $\xi=\{\nu, \phi\}$. It is known that the robit link with about 7 degrees of freedom provides an excellent approximation to the logit link. Thus Zhang's (2002) model is (approximately) part of our models to choose from.

The spatial random field is assumed to have constant mean $\beta$ and partial sill parameter $\sigma^{2}$ which are assigned the normal and scaled-inverse-chi-square priors of Section 3 with hyperparameter values $m_{b}=0, V_{b}=10, n_{\sigma}=4$, and $a_{\sigma}=10$. We also fix $\omega=0$ as we found that estimating this parameter along with the other parameters results in serious overfit to the data.

For each model we choose the skeleton set by the method described in Section 3.2 with $T=4$ and discard points that fall below $60 \%$ of the maximum marginal value. This procedure resulted in 8, 8, 9, and 9 skeleton points for the models in Table 6 respectively (see Appendix C, Table 9).

For each model, we generate Markov Chain samples of size given by $N / k$ (rounded down), where $N=50000$ and $k$ is the number of skeleton points for each model given in the previous paragraph, from $\pi_{\xi}\left(\beta, \sigma^{2}, \mathbf{z} \mid \mathbf{y}\right)$, after discarding a burn in of 300 samples, corresponding to each point $\xi$ in the skeleton set. We use approximately $80 \%$ of these samples for the reverse logistic regression estimation and the remaining $20 \%$ of the samples to form GIS estimators and estimate $\hat{\xi}$. These estimates are shown in Table 6 along with the posterior mean estimates of $\beta$ and $\sigma^{2}$ based on the density $\pi_{\hat{\xi}}\left(\beta, \sigma^{2}, \mathbf{z} \mid \mathbf{y}\right)$ using $5000 \mathrm{MC}$ samples from this density, after a burn-in of 300 samples. It can be seen that the chosen models are simplified versions of the more general fitted models. In the case of the robit link, the probit link is selected, and in the case of the modified GEV link with exponential correlation, the Gumbel (log-log) link is selected.

We calculate the weight of each model as discussed in Section 3.3 using the samples generated from $\pi_{\hat{\xi}}\left(\beta, \sigma^{2}, \mathbf{z} \mid \mathbf{y}\right)$. The estimates of the Bayes factors relative to the probit-spherical model are shown in Table 6. It can be seen that the modified GEV link and exponential correlation have higher weight than the robit link and spherical correlation respectively. For most models the estimate of the link function parameter is at the boundary of the parameter space so there is evidence that using a parameterized link function is overfitting the data. The estimated infection probability (posterior mean) is shown in Figure 7 from each model, along with the ensemble prediction and the observed proportion of infections. It can be seen that the models give similar predictions and the prediction pattern resembles that of Zhang (2002) and of the observed data.

A leave-one-out crossvalidation was performed to assess each model fitted as well as the ensemble model. For each model we calculate the average negative predictive score as with the example of Section 5.1 but using the binomial instead of the Poisson pmf, and the average RMSE. The results 

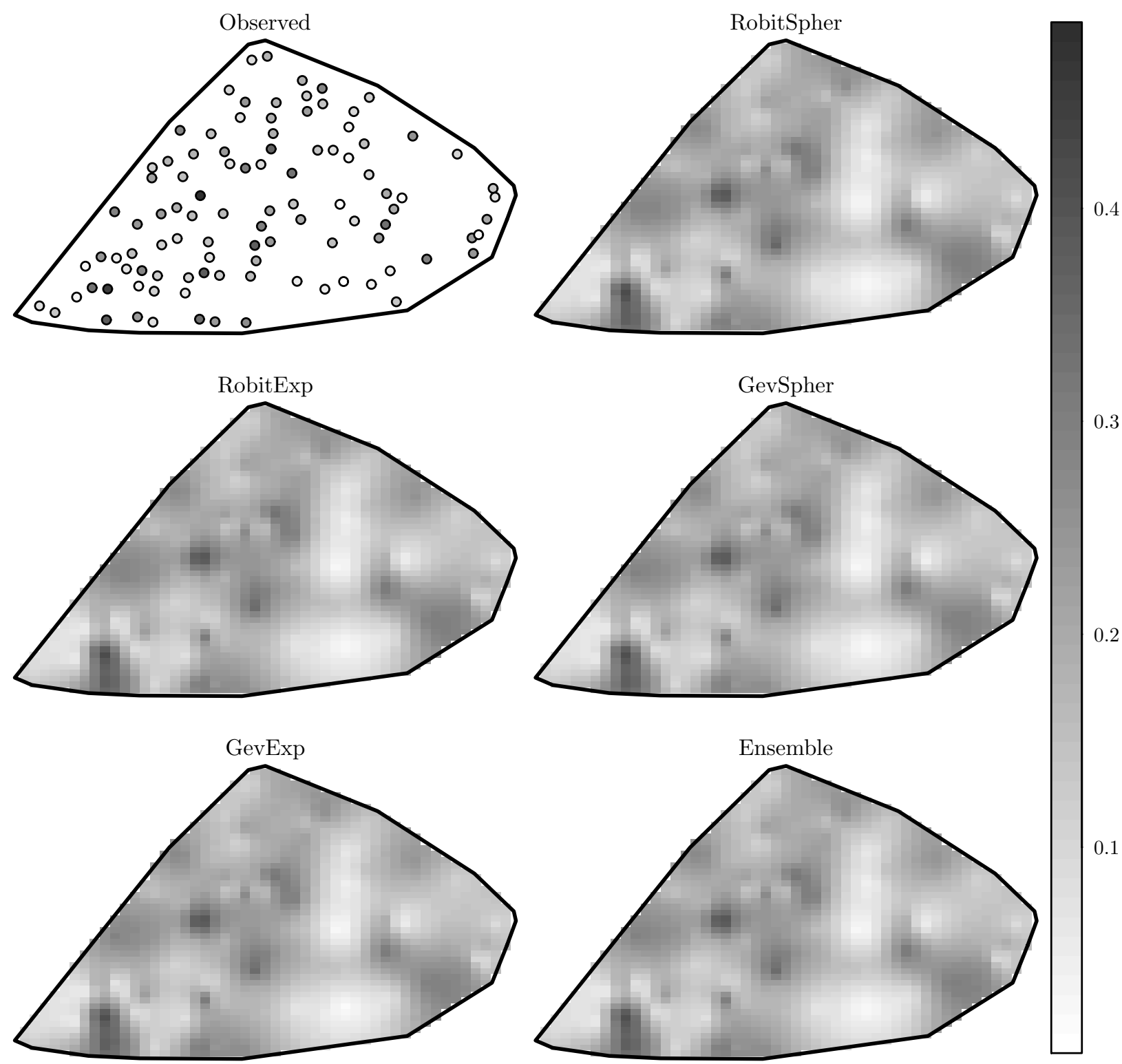

Figure 7: Observed proportion of infected roots and prediction of the binomial probability for the Rhizoctonia example under four different models and ensemble prediction. 


\begin{tabular}{lrrrrr}
\hline & Robit, Spher & Robit, Exp & M-GEV, Spher & M-GEV, Exp & Ensemble \\
\hline NegScore & 1367 & 1371 & 1373 & 1381 & 956 \\
RMSE & 207 & 207 & 201 & 202 & 167 \\
\hline
\end{tabular}

Table 7: Negative score and RMSE for the models used in the analysis of the rhizoctonia example. Smaller values are preferred.

are shown in Table 7 . The results are conflicting: the robit-spherical model is the best according to the negative score criterion and the modified-GEV-exponential the worse, but the modified-GEVspherical is the best according to the RMSE criterion and the robit-exponential the worse. There is a significant amount of variability which makes it difficult to make a proper assessment among the four models considered, however, it can be seen that the ensemble model, as with the previous example, is again significantly better using either measure.

\section{Conclusion and discussion}

In this paper we discuss SGLMMs where the link function contains unknown parameters. These models can be more robust compared to models which use a prescribed link function. Some of the proposed flexible link functions in the literature are not consistent with the Gaussian assumption of the latent spatial field, so we propose simple modifications to make them consistent without losing their flexibility. The central theme of the paper is the estimation of the link function and spatial correlation parameters by maximizing the Bayes factors relative to a fixed model. Therefore the methodology is developed around the ability to compute these Bayes factors efficiently. We show that naive generalized importance sampling estimation can sometimes fail, and show how by using suitable transformations to the samples can give accurate results. Thus we develop effective GIS and reverse logistic estimators based on appropriately chosen reparameterizations. The reparameterization is shown to reduce the variability in GIS estimators, and also alleviates the well-known separability problem of Geyer's (1994) reverse logistic regression estimator. We also use the RL method to compare models which have different families of link and correlation functions, thus providing a method of choosing and weighting different spatial models. This also allows for ensemble estimation and prediction of the mean response. In fact, for the two examples presented in this paper, the ensemble prediction outperforms predictions based on a single model.

The choice of importance sampling densities can impact the accuracy of the GIS estimators. In the context of the simple IS estimator, Botev et al. (2013) discuss construction of semi-parametric and nonparametric importance sampling densities using Markov chain samples (see also Beaujean and Caldwell, 2013). Here, we use Laplace approximation to marginal likelihoods for choosing suitable importance distributions for the GIS estimators. The new reparameterized GIS estimators and the EB methodology for selecting models, although developed in the context of SGLMMs here, are applicable to other models including generalized linear models and generalized linear mixed models. Also, the use of transformation can be similarly extended to improve other IS estimators, e.g. other multiple IS schemes (Veach and Guibas, 1995; Owen and Zhou, 2000; Elvira et al., 2015; Martino et al., 2017), parallel, serial or simulated tempering (George and Doss, 2018; Marinari and Parisi, 1992). Likewise, the proposed method of choosing importance densities for GIS can also be used for other IS estimators. 


\section{Appendices}

\section{A Detailed derivations}

\section{A.1 Standard errors for empirical Bayes estimates}

To estimate the variability in the empirical Bayes estimates for $\xi$, we compute

$$
\frac{\partial^{2}}{\partial \xi \partial \xi^{\top}} \log m_{\xi}(\mathbf{y})=\mathrm{E}\left(\frac{\partial^{2}}{\partial \xi \partial \xi^{\top}} \log p[\mathbf{y}, \mathbf{w} \mid \psi, \xi]\right)+\operatorname{Var}\left(\frac{\partial}{\partial \xi} \log p[\mathbf{y}, \mathbf{w} \mid \psi, \xi]\right),
$$

(see Casella, 2001) where the expectation and variance are taken with respect to the posterior density $\pi_{\xi}(\mathbf{w}, \psi \mid \mathbf{y})$.

To derive an explicit formula for our model, we write

$$
\log p\left[\mathbf{y} \mid \boldsymbol{\mu}=f_{\nu}\left(g_{\nu}(\mathbf{w})\right)\right]=\sum_{i=1}^{n}\left[\frac{1}{\chi}\left(y_{i} \gamma_{i}-t_{i} K\left(\gamma_{i}\right)\right)+c\left(y_{i}, \chi\right)\right],
$$

where $\gamma$ denotes the canonical parameter, $\chi$ is the dispersion parameter which is assumed known, $K(\gamma)$ is the cumulant function such that $K^{\prime}(\gamma)=\mu$, and $c(y, \chi)$ is a function which does not depend on $\gamma$ and not relevant to our analysis. For the binomial and Poisson models discussed here, $K(\gamma)=\log \left(1+e^{\gamma}\right)$ and $K(\gamma)=\exp (\gamma)$ respectively, and in both cases $\chi=1$ (McCullagh and Nelder, 1999). Specifically, we have the following relationship between $\gamma_{i}$ and $w_{i}, K^{\prime}\left(\gamma_{i}\right)=f_{\nu}\left(g_{\nu}\left(w_{i}\right)\right)$.

We also write $\vartheta=\{\theta, \omega\}$ for the covariance parameters and

$$
\log p\left[\mathbf{z}=g_{\nu}(\mathbf{w}) \mid \psi, \xi\right]=-\frac{1}{2 \sigma^{2}}(\mathbf{z}-X \beta)^{\top} R_{\vartheta}^{-1}(\mathbf{z}-X \beta)-\frac{1}{2} \log \left|R_{\vartheta}\right|-\frac{n}{2} \log \left(2 \pi \sigma^{2}\right),
$$

where $R_{\vartheta}$ denotes the matrix whose $(i, j)$ element is $\rho_{\theta}\left(\left\|s_{i}-s_{j}\right\|\right)+\omega I_{\left\{s_{i}=s_{j}\right\}}$ for sampling locations $s_{i}, s_{j}, i, j=1, \ldots, n$.

We now proceed to compute the necessary derivatives from (27) and (28). Note that

$$
\begin{aligned}
\frac{\partial}{\partial \nu} \log p[\mathbf{y} \mid \boldsymbol{\mu}] & =\frac{1}{\chi} \sum_{i=1}^{n}\left(y_{i}-t_{i} K^{\prime}\left(\gamma_{i}\right)\right) \frac{\partial \gamma_{i}}{\partial \nu} \\
\frac{\partial^{2}}{\partial \nu^{2}} \log p[\mathbf{y} \mid \boldsymbol{\mu}] & =\frac{1}{\chi} \sum_{i=1}^{n}\left(y_{i}-t_{i} K^{\prime}\left(\gamma_{i}\right)\right) \frac{\partial^{2} \gamma_{i}}{\partial \nu^{2}}-\frac{1}{\chi} \sum_{i=1}^{n} t_{i} K^{\prime \prime}\left(\gamma_{i}\right)\left(\frac{\partial \gamma_{i}}{\partial \nu}\right)^{2},
\end{aligned}
$$

where

$$
\begin{gathered}
K^{\prime \prime}\left(\gamma_{i}\right) \frac{\partial \gamma_{i}}{\partial \nu}=\frac{\partial}{\partial \nu} f_{\nu}\left(z_{i}\right) \cdot \frac{\partial}{\partial \nu} g_{\nu}\left(w_{i}\right) \\
K^{\prime \prime}\left(\gamma_{i}\right) \frac{\partial^{2} \gamma_{i}}{\partial \nu^{2}}+K^{\prime \prime \prime}\left(\gamma_{i}\right)\left(\frac{\partial \gamma_{i}}{\partial \nu}\right)^{2} \\
=\frac{\partial^{2}}{\partial \nu^{2}} f_{\nu}\left(z_{i}\right) \cdot\left(\frac{\partial}{\partial \nu} g_{\nu}\left(w_{i}\right)\right)^{2}+\frac{\partial}{\partial \nu} f_{\nu}\left(z_{i}\right) \cdot \frac{\partial^{2}}{\partial \nu^{2}} g_{\nu}\left(w_{i}\right) .
\end{gathered}
$$

From (28) we have

$$
\begin{aligned}
\frac{\partial}{\partial \nu} \log p[\mathbf{z} \mid \psi, \xi] & =-\frac{1}{\sigma^{2}}(\mathbf{z}-X \beta)^{\top} R_{\vartheta}^{-1}\left(\frac{\partial}{\partial \nu} g_{\nu}(\mathbf{w})\right) \\
\frac{\partial^{2}}{\partial \nu^{2}} \log p[\mathbf{z} \mid \psi, \xi] & =-\frac{1}{\sigma^{2}}\left(\frac{\partial}{\partial \nu} g_{\nu}(\mathbf{w})\right)^{\top} R_{\vartheta}^{-1} \frac{\partial}{\partial \nu} g_{\nu}(\mathbf{w})-\frac{1}{\sigma^{2}}(\mathbf{z}-X \beta)^{\top} R_{\vartheta}^{-1} \frac{\partial^{2}}{\partial \nu^{2}} g_{\nu}(\mathbf{w}) .
\end{aligned}
$$


We write $\partial_{j} R_{\vartheta}$ for the derivative of $R_{\vartheta}$ with respect to the $j$ th component of $\vartheta$ and similarly for higher-order derivatives. We have,

$$
\begin{aligned}
\frac{\partial}{\partial \vartheta_{j}} \log p[\mathbf{z} \mid \psi, \xi]= & \frac{1}{2 \sigma^{2}}(\mathbf{z}-X \beta)^{\top}\left(R_{\vartheta}^{-1} \partial_{j} R_{\vartheta} R_{\vartheta}^{-1}\right)(\mathbf{z}-X \beta)-\frac{1}{2} \operatorname{tr}\left(R_{\vartheta}^{-1} \partial_{j} R_{\vartheta}\right), \\
\frac{\partial^{2}}{\partial \vartheta_{j} \partial \vartheta_{k}} \log p[\mathbf{z} \mid \psi, \xi]= & \frac{1}{2 \sigma^{2}}(\mathbf{z}-X \beta)^{\top}\left(R_{\vartheta}^{-1} \partial_{j k}^{2} R_{\vartheta} R_{\vartheta}^{-1}\right)(\mathbf{z}-X \beta) \\
& -\frac{1}{\sigma^{2}}(\mathbf{z}-X \beta)^{\top}\left(R_{\vartheta}^{-1} \partial_{j} R_{\vartheta} R_{\vartheta}^{-1} \partial_{k} R_{\vartheta} R_{\vartheta}^{-1}\right)(\mathbf{z}-X \beta) \\
& +\frac{1}{2} \operatorname{tr}\left(R_{\vartheta}^{-1} \partial_{j} R_{\vartheta} R_{\vartheta}^{-1} \partial_{k} R_{\vartheta}\right)-\frac{1}{2} \operatorname{tr}\left(R_{\vartheta}^{-1} \partial_{j k}^{2} R_{\vartheta}\right) .
\end{aligned}
$$

Recall also the Jacobian term $\bar{J}_{\nu}(\mathbf{w})=\prod_{i=1}^{n} g_{\nu}^{\prime}\left(w_{i}\right)$. Therefore,

$$
\begin{aligned}
\frac{\partial}{\partial \nu} \log \bar{J}_{\nu}(\mathbf{w}) & =\sum_{i=1}^{n} \frac{1}{g_{\nu}^{\prime}\left(w_{i}\right)} \frac{\partial}{\partial \nu} g_{\nu}^{\prime}\left(w_{i}\right), \\
\frac{\partial^{2}}{\partial \nu^{2}} \log \bar{J}_{\nu}(\mathbf{w}) & =\sum_{i=1}^{n} \frac{1}{g_{\nu}^{\prime}\left(w_{i}\right)} \frac{\partial^{2}}{\partial \nu^{2}} g_{\nu}^{\prime}\left(w_{i}\right)-\sum_{i=1}^{n}\left(\frac{1}{g_{\nu}^{\prime}\left(w_{i}\right)} \frac{\partial}{\partial \nu} g_{\nu}^{\prime}\left(w_{i}\right)\right)^{2} .
\end{aligned}
$$

In practice we let $\mathcal{H}$ be the matrix $\mathcal{H}=-\frac{\partial^{2}}{\partial \xi \partial \xi^{\top}} \log m_{\hat{\xi}}(\mathbf{y})$ and $\hat{\mathcal{H}}$ be its Monte-Carlo approximation derived using samples $\left\{\mathbf{z}^{(l)}, \psi^{(l)}\right\}_{l=1}^{N}$ from the posterior density $\pi_{\hat{\xi}}(\mathbf{z}, \psi \mid \mathbf{y})$ (or equivalently using samples $\left\{g_{\hat{\nu}}^{-1}\left(\mathbf{z}^{(l)}\right), \psi^{(l)}\right\}_{l=1}^{N}$ from the posterior density $\left.\pi_{\hat{\xi}}(\mathbf{w}, \psi \mid \mathbf{y})\right)$. Then, we approximate the variance of $\hat{\xi}$ by $\hat{\mathcal{H}}^{-1}$.

\section{A.2 Laplace approximation}

We write the prior pdf for $\beta \mid \sigma^{2}, \pi\left(\beta \mid \sigma^{2}\right)$ as

$$
\log \pi\left(\beta \mid \sigma^{2}\right)=-\frac{1}{2 \sigma^{2}}\left(\beta-m_{b}\right)^{\top} V_{b}^{-1}\left(\beta-m_{b}\right)-\frac{1}{2} \log \left|V_{b}\right|-\frac{p}{2} \log \left(2 \pi \sigma^{2}\right) .
$$

Simple calculations show that integrating out $\beta, p\left[\mathbf{z} \mid \sigma^{2}, \xi\right]=\int_{\mathcal{R}^{p}} p\left[\mathbf{z} \mid \beta, \sigma^{2}, \xi\right] \pi\left(\beta \mid \sigma^{2}\right) \mathrm{d} \beta$ is given by

$$
\log p\left[\mathbf{z} \mid \sigma^{2}, \xi\right]=-\frac{1}{2 \sigma^{2}}\left(\mathbf{z}-X m_{b}\right)^{\top} T_{\vartheta}\left(\mathbf{z}-X m_{b}\right)+\frac{1}{2} \log \left|T_{\vartheta}\right|-\frac{n}{2} \log \left(2 \pi \sigma^{2}\right),
$$

where

$$
T_{\vartheta}=R_{\vartheta}^{-1}-R_{\vartheta}^{-1} X\left(V_{b}^{-1}+X^{\top} R_{\vartheta}^{-1} X\right)^{-1} X^{\top} R_{\vartheta}^{-1} .
$$

Then, from (27) and (29), we choose $\tilde{\mathbf{z}}$ such that

$$
\tilde{\mathbf{z}}=\tilde{\mathbf{z}}_{\xi}\left(\sigma^{2}\right)=\underset{\mathbf{z}}{\operatorname{argmax}} \log p[\mathbf{y} \mid \mathbf{z}, \xi]+\log p\left[\mathbf{z} \mid \sigma^{2}, \xi\right],
$$

which is straightforward to obtain using a quasi-Newton algorithm (Byrd et al., 1995).

The matrix $\tilde{H}_{\xi}\left(\sigma^{2}\right)$ in (22) is given by

$$
\tilde{H}_{\xi}\left(\sigma^{2}\right)=\frac{1}{\sigma^{2}} T_{\vartheta}+\frac{1}{\chi} \tilde{D}_{\vartheta},
$$

where $\chi$ is as in Appendix A.1 and

$$
\tilde{D}_{\vartheta}=\operatorname{diag}\left\{\left.t_{i} f_{\nu}^{\prime}\left(\tilde{z}_{i}\right) \frac{\partial \gamma_{i}}{\partial z_{i}}\right|_{z_{i}=\tilde{z}_{i}}-\left.\left(y_{i}-t_{i} f_{\nu}\left(\tilde{z}_{i}\right)\right) \frac{\partial^{2} \gamma_{i}}{\partial z_{i}^{2}}\right|_{z_{i}=\tilde{z}_{i}}\right\}_{i=1}^{n},
$$

which is used for the evaluation of the integrand in (23). 


\section{B Summary of the steps involved in inference}

In the proposed empirical Bayes formulation for SGLMMs, first, an estimate of $\hat{\xi}$ is found using one of the reparameterized GIS methods that involves the following two stages.

Stage 1 Draw MCMC samples $\left\{\psi^{(j ; l)}, \mathbf{z}^{(j ; l)}\right\}_{l=1}^{\tilde{N}_{j}}$ from $\pi_{\xi_{j}}(\psi, \mathbf{z} \mid \mathbf{y})$ for $j=1, \ldots, k$, and use these samples to estimate $\mathbf{r}$ by the reverse logistic regression method. For the log quasi likelihood function in the RL estimation, $p\left[\mathbf{y}, \mathbf{z} \mid \psi, \xi_{j}\right]$ can be replaced by either $p\left[\mathbf{z}=h_{\nu_{j}}(\boldsymbol{\mu}) \mid \psi, \xi_{j}\right] \tilde{J}_{\nu_{j}}(\boldsymbol{\mu})$ or $p\left[\mathbf{y} \mid \boldsymbol{\mu}=f_{\nu_{j}}\left(g_{\nu_{j}}(\mathbf{w})\right)\right] p\left[\mathbf{z}=g_{\nu_{j}}(\mathbf{w}) \mid \psi, \xi_{j}\right] \bar{J}_{\nu_{j}}(\mathbf{w})$ depending on whether the reparameterized samples are obtained using the transformation $h_{\nu}^{-1}: \mathbf{z} \mapsto \boldsymbol{\mu}$ or $g_{\nu}^{-1}: \mathbf{z} \mapsto \mathbf{w}$.

Stage 2 Independently of Stage 1, draw new MCMC samples $\left\{\psi^{(j ; l)}, \mathbf{z}^{(j ; l)}\right\}_{l=1}^{N_{j}}$ from $\pi_{\xi_{j}}(\psi, \mathbf{z} \mid \mathbf{y})$ for $j=1, \ldots, k$. Use these samples and $\hat{\mathbf{r}}$ computed in Stage 1 to estimate the BFs $B_{\xi, \xi_{1}}$ by either of the two proposed reparameterized GIS estimators $\tilde{B}_{\xi, \xi_{1}}(\tilde{\mathbf{r}})$ (given in (15)) or $\bar{B}_{\xi, \xi_{1}}(\overline{\mathbf{r}})$ (given in (17)).

Estimate $\hat{\xi}$ by maximizing either $\tilde{B}_{\xi, \xi_{1}}(\tilde{\mathbf{r}})$ or $\bar{B}_{\xi, \xi_{1}}(\overline{\mathbf{r}})$.

After finding the EB estimate $\hat{\xi}$, draw new MCMC samples $\left\{\psi^{(i)}, \mathbf{z}^{(i)}\right\}_{i=1}^{M}$ from $\pi_{\hat{\xi}}(\psi, \mathbf{z} \mid \mathbf{y})$ to make inference on $\psi$ as well as the latent Gaussian random field $\{Z(s), s \in \mathbb{S}\}$. If multiple families of link functions (and/or covariance functions) are under consideration, then the ensemble estimates given in (26) can be used to make inference on $\psi$ and the random field.

\section{List of skeleton points used in the examples}

This section lists the skeleton set obtained using the method of Section 3.2 for the examples of Section 5.1 (Table 8) and Section 5.2 (Table 9).

\begin{tabular}{|c|c|c|c|c|c|c|c|c|c|c|c|c|c|}
\hline \multicolumn{4}{|c|}{ Matérn } & \multicolumn{4}{|c|}{ Exp-power } & \multicolumn{3}{|c|}{ Spherical } & \multicolumn{3}{|c|}{ Exponential } \\
\hline$\nu$ & $\phi$ & $\omega$ & $\kappa$ & $\nu$ & $\phi$ & $\omega$ & $\kappa$ & $\nu$ & $\phi$ & $\omega$ & $\nu$ & $\phi$ & $\omega$ \\
\hline 0.94 & 415 & 0.970 & 0.28 & 0.96 & 140 & 0.770 & 0.410 & 0.97 & 660 & 2.65 & 0.96 & 580 & 2.4 \\
\hline 0.94 & 700 & 0.970 & 0.28 & 0.96 & 720 & 0.770 & 0.410 & 0.97 & 1130 & 2.65 & 1.10 & 580 & 2.4 \\
\hline 0.94 & 415 & 2.385 & 0.28 & 0.96 & 1300 & 0.770 & 0.410 & 1.10 & 1130 & 2.65 & 0.96 & 980 & 2.4 \\
\hline 0.94 & 700 & 2.385 & 0.28 & 0.96 & 720 & 2.035 & 1.005 & 0.97 & 1600 & 2.65 & 0.96 & 580 & 3.8 \\
\hline 1.10 & 700 & 2.385 & 0.28 & 1.10 & 720 & 2.035 & 1.005 & 0.97 & 1130 & 4.30 & & & \\
\hline 0.94 & 130 & 2.385 & 0.94 & 0.96 & 720 & 3.300 & 1.005 & & & & & & \\
\hline 0.94 & 415 & 2.385 & 0.94 & 1.10 & 720 & 3.300 & 1.005 & & & & & & \\
\hline 1.10 & 415 & 2.385 & 0.94 & 0.96 & 720 & 2.035 & 1.600 & & & & & & \\
\hline 0.94 & 415 & 3.800 & 0.94 & 0.96 & 720 & 3.300 & 1.600 & & & & & & \\
\hline 1.10 & 415 & 3.800 & 0.94 & 1.10 & 720 & 3.300 & 1.600 & & & & & & \\
\hline 0.94 & 130 & 2.385 & 1.60 & & & & & & & & & & \\
\hline 0.94 & 130 & 3.800 & 1.60 & & & & & & & & & & \\
\hline
\end{tabular}

Table 8: Skeleton set for Section 5.1 . 


\begin{tabular}{|c|c|c|c|c|c|c|c|}
\hline \multicolumn{2}{|c|}{ Robit, spherical } & \multicolumn{2}{|c|}{ Robit, exponential } & \multicolumn{2}{|c|}{ Mod GEV, spherical } & \multicolumn{2}{|c|}{ Mod GEV, exponentia } \\
\hline$\nu$ & $\phi$ & $\nu$ & $\phi$ & $\nu$ & $\phi$ & $\nu$ & $\phi$ \\
\hline 12 & 3300 & 40 & 990 & 0.12 & 2400 & 0.12 & 1400 \\
\hline 21 & 3300 & 21 & 1960 & 0.23 & 2400 & 0.25 & 1400 \\
\hline 31 & 3300 & 30 & 1960 & 0.35 & 2400 & 0.37 & 1400 \\
\hline 40 & 3300 & 40 & 1960 & 0.00 & 4667 & 0.00 & 2767 \\
\hline 21 & 4900 & 21 & 2930 & 0.12 & 4667 & 0.12 & 2767 \\
\hline 31 & 4900 & 30 & 2930 & 0.23 & 4667 & 0.25 & 2767 \\
\hline 40 & 4900 & 40 & 2930 & 0.00 & 6933 & 0.00 & 4133 \\
\hline \multirow[t]{2}{*}{40} & 6500 & 40 & 3900 & 0.12 & 6933 & 0.12 & 4133 \\
\hline & & & & 0.00 & 9200 & 0.00 & 5500 \\
\hline
\end{tabular}

Table 9: Skeleton set for Section 5.2 .

\section{Acknowledgment}

The authors thank two anonymous reviewers and an editor for several helpful comments and suggestions that led to a substantially improved revision of the paper.

\section{References}

Aranda-Ordaz, F. J. (1981). On two families of transformations to additivity for binary response data. Biometrika, 68(2):357.

Barndorff-Nielsen, O. E. and Cox, D. R. (1989). Asymptotic techniques for use in statistics. Chapman \& Hall.

Basu, A. and Rathouz, P. J. (2005). Estimating marginal and incremental effects on health outcomes using flexible link and variance function models. Biostatistics, 6(1):93-109.

Bazán, J. L., Branco, M. D., and Bolfarine, H. (2006). A skew item response model. Bayesian Analysis, 1(4):861-892.

Beaujean, F. and Caldwell, A. (2013). Initializing adaptive importance sampling with markov chains. arXiv preprint arXiv:1304.7808.

Berger, J. O., De Oliveira, V., and Sansó, B. (2001). Objective Bayesian analysis of spatially correlated data. Journal of the American Statistical Association, 96:1361-1374.

Botev, Z. I., L'Ecuyer, P., and Tuffin, B. (2013). Markov chain importance sampling with applications to rare event probability estimation. Statistics and Computing, 23(2):271-285.

Buckland, S. T., Burnham, K. P., and Augustin, N. H. (1997). Model selection: an integral part of inference. Biometrics, pages 603-618.

Buta, E. and Doss, H. (2011). Computational approaches for empirical Bayes methods and Bayesian sensitivity analysis. The Annals of Statistics, 39(5):2658-2685.

Byrd, R. H., Lu, P., Nocedal, J., and Zhu, C. (1995). A limited memory algorithm for bound constrained optimization. SIAM Journal on Scientific Computing, 16(5):1190-1208. 
Casella, G. (2001). Empirical Bayes Gibbs sampling. Biostatistics, 2(4):485-500.

Chen, M.-H., Dey, D. K., and Shao, Q.-M. (1999). A new skewed link model for dichotomous quantal response data. Journal of the American Statistical Association, 94(448):1172-1186.

Christensen, O. F. (2004). Monte Carlo maximum likelihood in model based geostatistics. Journal of Computational and Graphical Statistics, 13:702-718.

Christensen, O. F., Roberts, G. O., and Sköld, M. (2006). Robust Markov chain Monte Carlo methods for spatial generalized linear mixed models. Journal of Computational and Graphical Statistics, 15:1-17.

Christensen, O. F. and Waagepetersen, R. (2002). Bayesian prediction of spatial count data using generalized linear mixed models. Biometrics, 58:280-286.

Cran, G. W., Martin, K. J., and Thomas, G. E. (1977). Remark AS R19 and algorithm AS 109: A remark on algorithms: AS 63: The incomplete beta integral AS 64: Inverse of the incomplete beta function ratio. Journal of the Royal Statistical Society. Series C (Applied Statistics), 26(1):111114.

Diggle, P. J., Ribeiro, P. J., and Christensen, O. F. (2003). An introduction to model-based geostatistics. In Spatial statistics and computational methods. Lecture notes in statistics, pages 43-86. Springer.

Diggle, P. J., Tawn, J. A., and Moyeed, R. A. (1998). Model-based geostatistics. Journal of the Royal Statistical Society. Series C (Applied Statistics), 47:299-350.

Doss, H. (2010). Estimation of large families of Bayes factors from Markov chain output. Statistica Sinica, 20:537-560.

Doss, H. (2012). Hyperparameter and model selection for nonparametric Bayes problems via RadonNikodym derivatives. Statistica Sinica, 22:1-26.

Elvira, V., Martino, L., Luengo, D., and Bugallo, M. F. (2015). Generalized multiple importance sampling. ArXiv.

Evangelou, E. and Roy, V. (2018). geoBayes. R package version 0.5.

George, C. P. and Doss, H. (2018). Principled selection of hyperparameters in the latent Dirichlet allocation model. Journal of Machine Learning Research, 18(162):1-38.

Geyer, C. J. (1994). Estimating normalizing constants and reweighting mixtures in Markov chain Monte Carlo. Technical Report 568, School of Statistics, University of Minnesota.

Geyer, C. J. (1996). Markov chain Monte Carlo In Practice, chapter Estimation and optimization of functions, pages 241-258. Chapman and Hall/CRC Press, Boca Raton, FL.

Geyer, C. J. and Thompson, E. A. (1992). Constrained Monte Carlo maximum likelihood for dependent data. Journal of the Royal Statistical Society. Series B (Methodological), pages 657699.

Guerrero, V. M. and Johnson, R. A. (1982). Use of the Box-Cox transformation with binary response models. Biometrika, 69(2):309-314. 
Johnson, N. L., Kotz, S., and Balakrishnan, N. (1995). Continuous Univariate Distributions, volume 2 of Wiley series in probability and mathematical statistics. John Wiley \& Sons, New York.

Koenker, R. (2006). Parametric links for binary response. R News, 6(4):32-34.

Koenker, R. and Yoon, J. (2009). Parametric links for binary choice models: A Fisherian-Bayesian colloquy. Journal of Econometrics, 152(2):120-130.

Liu, C. (2004). Robit regression: A simple robust alternative to logistic and probit regression. In Gelman, A. and Meng, X. L., editors, Applied Bayesian Modeling and Casual Inference from Incomplete-Data Perspectives, pages 227-238. Wiley, London.

Liu, J. S. and Wu, Y. N. (1999). Parameter expansion for data augmentation. Journal of the American Statistical Association, 94:1264-1274.

Mardia, K. V. and Watkins, A. J. (1989). On multimodality of the likelihood in the spatial linear model. Biometrika, 76(2):289-295.

Marinari, E. and Parisi, G. (1992). Simulated tempering: A new Monte Carlo scheme. Europhysics Letters, 19:451-458.

Martino, L., Elvira, V., Luengo, D., and Corander, J. (2017). Layered adaptive importance sampling. Statistics and Computing, 27(3):599-623.

McCullagh, P. and Nelder, J. A. (1999). Generalized Linear Models. Chapman \& Hall Ltd.

Nagler, J. (1994). Scobit: an alternative estimator to logit and probit. American Journal of Political Science, pages 230-255.

Owen, A. and Zhou, Y. (2000). Safe and effective importance sampling. Journal of the American Statistical Association, 95:135-143.

Prentice, R. L. (1976). A generalization of the probit and logit methods for dose response curves. Biometrics, pages 761-768.

Roy, V. (2014). Efficient estimation of the link function parameter in a robust Bayesian binary regression model. Computational Statistics and Data Analysis, 73:87-102.

Roy, V., Evangelou, E., and Zhu, Z. (2015). Empirical bayes methods for the transformed gaussian random fields model with additive measurement errors. In Upadhyay, S. K., Singh, U., Dey, D. K., and Loganathan, A., editors, Current Trends in Bayesian Methodology with Applications, pages 521-536. Chapman \& Hall/CRC Press.

Roy, V., Evangelou, E., and Zhu, Z. (2016). Efficient estimation and prediction for the Bayesian binary spatial model with flexible link functions. Biometrics, 72(1):289-298.

Roy, V., Tan, A., and Flegal, J. (2018). Estimating standard errors for importance sampling estimators with multiple Markov chains. Statistica Sinica, 28(2):1079-1101.

Simpson, M., Niemi, J., and Roy, V. (2017). Interweaving Markov chain Monte Carlo strategies for efficient estimation of dynamic linear models. Journal of Computational and Graphical Statistics, $26: 152-159$.

Stein, M. L. (1999). Interpolation of Spatial Data. Springer Verlag, New York. 
Stukel, T. A. (1988). Generalized logistic models. Journal of the American Statistical Association, 83(402):426-431.

van Dyk, D. A. and Meng, X.-L. (2001). The art of data augmentation (with discussion). Journal of Computational and Graphical Statistics, 10:1-50.

Veach, E. and Guibas, L. (1995). Optimally combining sampling techniques for Monte Carlo rendering. SIGGRAPH 95 Conference Proceedings, Reading MA. Addison-Wesley, pages 419-428.

Wallace, D. L. (1959). Bounds on normal approximations to Student's and the chi-square distributions. The Annals of Mathematical Statistics, 30(4):1121-1130.

Wang, X. and Dey, D. K. (2010). Generalized extreme value regression for binary response data: An application to B2B electronic payments system adoption. The Annals of Applied Statistics, pages 2000-2023.

Wang, X., Dey, D. K., and Banerjee, S. (2010). Non-Gaussian hierarchical generalized linear geostatistical model selection. In Chen, M. H., Dey, D. K., Müller, P., Sun, D., and Ye, K., editors, Frontiers of Statistical Decision Making and Bayesian Analysis, pages 484-496. Springer.

Zhang, H. (2002). On estimation and prediction for spatial generalized linear mixed models. Biometrics, 58:129-136. 